MATHEMATICS OF COMPUTATION

Volume 76, Number 259, July 2007, Pages 1393-1424

S 0025-5718(07)01963-1

Article electronically published on February 5, 2007

\title{
ON THE PROBABILITY DISTRIBUTION OF CONDITION NUMBERS OF COMPLETE INTERSECTION VARIETIES AND THE AVERAGE RADIUS OF CONVERGENCE OF NEWTON'S METHOD IN THE UNDERDETERMINED CASE
}

\author{
C. BELTRÁN AND L. M. PARDO
}

\begin{abstract}
In these pages we show upper bound estimates on the probability distribution of the condition numbers of smooth complete intersection algebraic varieties. As a by-product, we also obtain lower bounds for the average value of the radius of Newton's basin of attraction in the case of positive dimension affine complex algebraic varieties.
\end{abstract}

\section{INTRODUCTION}

In these pages we prove several upper bound estimates concerning the average value of the quantities that dominate the computational behavior of Newton's operator in the underdetermined case. Newton's operator for underdetermined systems of equations was introduced by Shub and Smale in [SS96] (cf. also [Ded06). Their main goal was the design and analysis of efficient algorithms that compute approximations to complete intersection algebraic subvarieties of $\mathbb{C}^{n}$.

This introduction is devoted to motivating and stating the main outcomes of this paper. In order to be precise in our statements we need to introduce some preliminary notation. Let $l \in \mathbb{N}$ be a positive integer number. We denote by $P_{l}$ the complex vector space of all complex polynomials $f \in \mathbb{C}\left[X_{1}, \ldots, X_{n}\right]$ of degree at most $l$. For every list of positive degrees $(d):=\left(d_{1}, \ldots, d_{m}\right), m \leq n$, we denote by $\mathcal{P}_{(d)}^{m}$ the complex vector space given as the cartesian product

$$
\mathcal{P}_{(d)}^{m}:=\prod_{i=1}^{m} P_{d_{i}}
$$

Namely, $\mathcal{P}_{(d)}^{m}, m \leq n$, is the space of underdetermined systems of multivariate polynomial equations. We denote by $d$ the maximum of the degrees and by $\mathcal{D}$ the Bézout number associated with the list $(d)$. Namely,

$$
d:=\max \left\{d_{1}, \ldots, d_{m}\right\}, \quad \mathcal{D}:=\prod_{i=1}^{m} d_{i}
$$

Received by the editor February 6, 2006.

2000 Mathematics Subject Classification. Primary 65G50, 65H10.

Key words and phrases. Condition number, geometric integration theory.

This research was partially supported by MTM2004-01167 and FPU program, Government of Spain.

(C)2007 American Mathematical Society 
As in Kun85, a set-theoretical complete intersection affine algebraic variety of $\mathbb{C}^{n}$ of codimension $m$ is an algebraic subset $V \subseteq \mathbb{C}^{n}$ of dimension $n-m$ such that there is a degree list $(d)$ and some system of multivariate complex polynomials $f=\left[f_{1}, \ldots, f_{m}\right] \in \mathcal{P}_{(d)}^{m}$ satisfying

$$
V=V(f)=\left\{x \in \mathbb{C}^{n}: f_{i}(x)=0,1 \leq i \leq m\right\} .
$$

The case $m=n$ is simply the case of zero-dimensional complete intersection algebraic varieties.

A central computational problem is the design of efficient algorithms that solve the following problem.

\section{Approximating Complete Intersection Varieties}

\section{INPUT:}

- A list of polynomial equations $f=\left[f_{1}, \ldots, f_{m}\right] \in \mathcal{P}_{(d)}^{m}$ such that $V(f)$ is a complete intersection of codimension $m$.

- Some positive real number $\varepsilon>0$.

Output: A point $z \in \mathbb{C}^{n}$ in the tube of radius $\varepsilon$ about $V(f)$. Namely, a point $z \in \mathbb{C}^{n}$ such that

$$
\operatorname{dist}(z, V(f)):=\inf \{\|z-\zeta\|: \zeta \in V(f)\}<\varepsilon .
$$

The zero-dimensional case $(m=n)$ has been extensively studied in the series of deep papers by M. Shub and S. Smale [SS93b, SS93a, SS93c, SS94, SS96] (cf. also [Ded01, DS01, Kim89, GLSY05, Mal94]). For recent advances in this case, see BP06b.

Shub and Smale showed that the design of efficient algorithms for the zerodimensional case is a consequence of the knowledge of the average behavior of certain quantities associated with the input system $f \in \mathcal{P}_{(d)}^{m}$ that dominate Newton's operator (cf. [SS93a, Main Theorem] and also [BP06b). The aim of these pages is to contribute to Shub and Smale's program in the case of positive dimension solution varieties.

With the same notation as above, let $f \in \mathcal{P}_{(d)}^{m}$ be a system of polynomial equations and let $z \in \mathbb{C}^{n}$ be a complex point. Newton's operator of $f$ at $z$ is defined by the following identity:

$$
N_{f}(z):=z-\left(d_{z} f\right)^{\dagger} f(z),
$$

where $d_{z} f$ is the Jacobian matrix of $f$ at $z$ and $\left(d_{z} f\right)^{\dagger}$ is the Moore-Penrose pseudoinverse of $d_{z} f$.

With this notation, a point $z$ is called an approximate zero of $f$ if the sequence of iterations of Newton's operator applied to $z$ is convergent and for every positive integer number $k \in \mathbb{N}$ the following inequality holds:

$$
\operatorname{dist}\left(N_{f}^{k}(z), V(f)\right) \leq \frac{2}{2^{2^{k}-1}} \operatorname{dist}(z, V(f)),
$$

where $\operatorname{dist}(\cdot, V(f))$ denotes distance to the algebraic variety $V(f)$ (we say that the speed of convergence is doubly exponential). Let the reader observe that our definition also implies there will be a point $\zeta \in V(f)$ such that $N_{f}^{k}(z)$ converges to 
$\zeta$. Moreover, in all usages below (i.e., under the $\gamma$-theorem's hypothesis) the speed of this convergence will also be doubly exponential.

Within Shub and Smale's program, the problem of approximating complete intersection varieties can be decomposed into two main steps:

- First, compute some approximate zero $z \in \mathbb{C}^{n}$.

- Then, apply Newton's operator to approximate a point $\zeta \in V(f)$.

The convergence of Newton's operator (in the underdetermined case) at a point is granted by the $\gamma$-theorem proved in [SS96] (cf. also Ded06] or Theorem 2.1] in Section 2.1). This $\gamma$-theorem introduces a quantity $\gamma(f, \zeta)$ depending on the input system $f \in \mathcal{P}_{(d)}^{m}$ and a regular solution $\zeta \in V(f)$, as follows:

$$
\gamma(f, x):=\sup _{k \geq 2}\left\|\left(d_{\zeta} f\right)^{\dagger} \frac{d_{\zeta}^{(k)} f}{k !}\right\|_{2}^{\frac{1}{k-1}},
$$

where $d_{\zeta}^{(k)} f$ is the $k$-th derivative of $f$ at $\zeta$, considered as a $k$-multilinear map. If $\zeta$ is not a regular solution, we define $\gamma(f, \zeta)=+\infty$. Here we strengthen this notion by introducing a maximum value for $\gamma$. Namely, for $f \in \mathcal{P}_{(d)}^{m}$ we define

$$
\gamma_{\text {worst }}(f):=\sup _{\zeta \in V(f)} \gamma(f, \zeta),
$$

and we prove the following statement, which is a corollary of Theorem C1 in SS96] or [Ded06, th. 134] (cf. Section 2.1 for a proof of this statement).

Corollary 1.1. There exists a universal constant $u_{0}, 0<u_{0} \sim 0.05992$ such that the following holds: Let $z \in \mathbb{C}^{n}$ be an affine point such that

$$
\operatorname{dist}(z, V(f)) \leq \frac{u_{0}}{\gamma_{\text {worst }}(f)} .
$$

Then, $z$ is an approximate zero of $f \in \mathcal{P}_{(d)}^{m}$.

The bottleneck of this result is that $\gamma_{\text {worst }}(f)$ may be infinite. For instance, if $V(f)$ contains some critical point $\zeta$ of $f: \mathbb{C}^{n} \longrightarrow \mathbb{C}^{m}$, then $\gamma(f, \zeta)=+\infty$ and $\gamma_{\text {worst }}(f)=+\infty$. However, we will see that for most systems $f$ the number $\gamma_{\text {worst }}(f)$ is finite. More precisely, assume that $\mathcal{P}_{(d)}^{m}$ is endowed with the Gaussian probability distribution with respect to the Bombieri-Weyl Hermitian product (see Section 2) and let $N+1$ be the complex dimension of $\mathcal{P}_{(d)}^{m}$. Then, we prove the following statement in Section 6.2.

Theorem 1.2. With the notation above, the following properties hold:

(1) $\gamma_{\text {worst }}(f)<+\infty$ almost everywhere in $\mathcal{P}_{(d)}^{m}$. Namely,

$$
\operatorname{Prob}\left[f \in \mathcal{P}_{(d)}^{m}: \gamma_{\text {worst }}(f)<+\infty\right]=1 .
$$

(2) The expectation of $\gamma_{\text {worst }}$ is bounded by the following inequality:

$$
\mathrm{E}_{\mathcal{P}_{(d)}^{m}}\left[\gamma_{\text {worst }}\right] \leq \frac{\mathcal{D}^{1 / 4}}{2}\left[10 m \sqrt{n N} d^{3 / 2}\right]^{\frac{n-m+2}{2}} .
$$

(3) The expectation of the convergence radius $\frac{u_{0}}{\gamma_{\text {worst }}}$ is bounded by the following inequality:

$$
\mathrm{E}_{\mathcal{P}_{(d)}^{m}}[(\text { Conv. radius })] \geq \frac{2 u_{0}}{\mathcal{D}^{1 / 4}\left[10 m \sqrt{n N} d^{3 / 2}\right]^{\frac{n-m+2}{2}}} .
$$


This theorem then means the following. For almost all complete intersection affine algebraic varieties $V \subseteq \mathbb{C}^{n}$, there is a nontrivial tube $V_{R}$ about $V$ of radius $R>0$ such that all points of $V_{R}$ are approximate zeros in the above sense. Moreover, claim (3) provides a lower bound for the average value of the radius $R$ of this tube.

Theorem 1.2 is a consequence of the study of the probability distribution of another quantity associated with the input system $f \in \mathcal{P}_{(d)}^{m}$ : the condition number $\mu_{\text {norm }}^{m}(f, \zeta)$, for $\zeta \in V(f)$ (see identity (2.2)). This quantity is strongly related to the $\mu_{\text {norm }}$ introduced in [SS93b, SS93a, Dég01.

This condition number $\mu_{\text {norm }}^{m}(f, \zeta)$, for $\zeta \in V(f)$, has two main properties. Firstly, in the zero-dimensional case, it is an upper bound for the complexity of procedures based on homotopic deformation techniques that approximate zerodimensional algebraic varieties. Secondly, in the underdetermined case it has been shown to control the stability of the solution set. For these results, see SS93a, SS94, Ded97, BP06b, Dég01. Moreover, in Proposition 3.4 we prove that for $f \in \mathcal{P}_{(d)}^{m}$, $\zeta \in V(f)$ the following inequality holds:

$$
\gamma(f, \zeta) \leq \frac{d^{3 / 2}}{2} \mu_{\text {norm }}^{m}(f, \zeta)
$$

just by arguments analogous to those used for the zero-dimensional case in SS93a.

Here we also contribute to Shub and Smale's program by studying the probability distribution of $\mu_{\text {norm }}^{m}$ in the positive dimension case. We study two variations of the condition number $\mu_{\text {norm }}^{m}$.

First we define the worst case condition number of an input system $f \in \mathcal{P}_{(d)}^{m}$ in its variety of zeros $V(f) \subseteq \mathbb{P}_{n}(\mathbb{C})$. Namely,

$$
\mu_{\mathrm{worst}}^{m}(f):=\sup _{\zeta \in V(f)} \mu_{\text {norm }}^{m}(f, \zeta)
$$

Then, we prove the following statement.

Theorem 1.3. Let $(d)=\left(d_{1}, \ldots, d_{m}\right)$ be such that $d_{i}>1$ for some $i, 1 \leq i \leq m$. Then, the following properties hold:

- $\mu_{\mathrm{worst}}^{m}(f)<+\infty$ almost everywhere in $\mathcal{P}_{(d)}^{m}$. Namely,

$$
\operatorname{Prob}\left[f \in \mathcal{P}_{(d)}^{m}: \mu_{\mathrm{worst}}^{m}(f)<+\infty\right]=1 .
$$

- The expectation in $\mathcal{P}_{(d)}^{m}$ of the worst-case condition number $\mu_{\mathrm{worst}}^{m}$ is bounded by the following inequality:

$$
\mathrm{E}_{\mathcal{P}_{(d)}^{m}}\left[\mu_{\text {worst }}^{m}\right] \leq \frac{\mathcal{D}^{1 / 4}}{d^{3 / 2}}\left[10 m \sqrt{n N} d^{3 / 2}\right]^{\frac{n-m+2}{2}} .
$$

Then, Theorem 1.2 is an almost immediate consequence of equation (1.2) and Theorem 1.3. Hence, we will concentrate our efforts on the proof of Theorem 1.3

Moreover, the use of a uniform tube about a complete intersection affine algebraic variety $V \subseteq \mathbb{C}^{n}$ is probably insufficient to explain the behavior and efficiency of Newton's operator in the underdetermined case. For this reason we also study the average behavior of $\mu_{\text {norm }}^{m}(f, \zeta)$ when $\zeta$ runs over the points in $V(f)$.

Although we have used the condition number $\mu_{\text {norm }}^{m}$ to estimate an affine radius, it is by nature (i.e., as all useful condition numbers) a projective function. Thus, we will analyze the average value of $\mu_{\text {norm }}^{m}$ as follows. Let $\mathbb{P}_{n}(\mathbb{C})$ be the $n$-dimensional complex projective space. For every system $f \in \mathcal{P}_{(d)}^{m}$, let $V_{\mathbb{P}}(f) \subseteq \mathbb{P}_{n}(\mathbb{C})$ be the projective closure of $V(f)$ for the Zariski topology in $\mathbb{P}_{n}(\mathbb{C})$. 
Assume now that $V_{\mathbb{P}}(f)$ is a complex smooth submanifold of $\mathbb{P}_{n}(\mathbb{C})$. Then it is endowed with a complex Riemannian structure that induces a volume form and a probability distribution in a natural way. Then, for every $f \in \mathcal{P}_{(d)}^{m}$ such that $V_{\mathbb{P}}(f)$ is smooth we define $\mu_{\mathrm{av}}^{m}(f)$ as the average value of $\mu_{\text {norm }}^{m}$ at $\zeta \in V_{\mathbb{P}}(f)$. Namely,

$$
\mu_{\mathrm{av}}^{m}(f):=\mathrm{E}_{\zeta \in V_{\mathbb{P}}(f)}\left[\mu_{\mathrm{norm}}^{m}(f, \zeta)\right] .
$$

In the case that $V_{\mathbb{P}}(f)$ contains some singularity we define $\mu_{\mathrm{av}}^{m}(f):=+\infty$.

Note that $\mu_{\mathrm{av}}^{m}(f)$ controls in some sense the expected stability of the solution set $V_{\mathbb{P}}(f)$. Then we also prove the following statement in Section 5 below.

Theorem 1.4. Let $(d)=\left(d_{1}, \ldots, d_{m}\right)$ be such that $d_{i}>1$ for some $i, 1 \leq i \leq m$. Then, the expected value of the condition number $\mu_{\mathrm{av}}^{m}$ satisfies

$$
\mathrm{E}_{\mathcal{P}_{(d)}^{m}}\left[\mu_{\mathrm{av}}^{m}\right] \leq 3 m \sqrt{n N}
$$

In the case that $m=1$, we can even obtain an equality (cf. Theorem 5.1). As a main outcome of Theorem 1.4 we observe that the average value of the condition number $\mu_{\text {norm }}^{m}$ of a complete intersection algebraic variety is much better behaved than its worst case estimate. This of course means that, for a randomly chosen system $f \in \mathcal{P}_{(d)}$, we can expect most parts of the variety $V_{\mathbb{P}}(f)$ to be very stable in the sense of Dég01.

This paper is structured as follows. Section 2 is devoted to stating a precise definition of the notions used in this Introduction and some other technical results. Section 3 is devoted to proving inequality (1.2). In Section 4 we prove the main technical tool for integration of functions in the set of systems. In Section 5 we prove Theorem 1.4 and in Section 6 we prove Theorems 1.3 and 1.2 .

\section{Preliminary Results}

2.1. Background. Recall the following theorem from Ded06] (cf. also SS96]).

Theorem 2.1. Let $f \in \mathcal{P}_{(d)}^{m}$ be a polynomial system, and let $\mathcal{V}_{f}$ be the following set:

$$
\mathcal{V}_{f}:=\left\{x \in \mathbb{C}^{n}: \exists \zeta \in V(f),\|x-\zeta\| \gamma(f, \zeta) \leq u_{0}\right\}
$$

where $u_{0}$ is a universal constant (about 0.05992). Let $x \in \mathcal{V}_{f}$ be a point, and let $\zeta \in \mathbb{C}^{n}$ be a solution of $f$ such that $\|x-\zeta\| \gamma(f, \zeta) \leq u_{0}$. Then, the Newton series $x_{k}:=N_{f}^{k}(x)$ converges to a point $\zeta^{\prime} \in V(f)$, and the following inequality holds for every $k \geq 0$ :

$$
\left\|x_{k}-\zeta^{\prime}\right\| \leq \frac{2}{2^{2^{k}-1}}\|x-\zeta\|
$$

Observe that, for any $f \in \mathcal{P}_{(d)}^{m}$, the set $\mathcal{V}_{f}$ is a "tubular" neighborhood of the solution set of $f$, and the "radius" of this neighborhood at each solution point $\zeta$ is exactly

$$
\frac{u_{0}}{\gamma(f, \zeta)}
$$

2.1.1. Proof of Corollary 1.1. Let $\zeta \in V(f)$ be such that $\operatorname{dist}(x, V(f))=\|x-\zeta\|$. Then, the following chain of inequalities holds:

$$
\|x-\zeta\| \gamma(f, \zeta) \leq \operatorname{dist}(x, V(f)) \gamma_{\text {worst }}(f) \leq u_{0}
$$


From Theorem 2.1, there exists a solution $\zeta^{\prime}$ of $f$ such that the Newton series $x_{k}:=N_{f}^{k}(x)$ satisfies

$$
\operatorname{dist}\left(x_{k}, V(f)\right) \leq\left\|x_{k}-\zeta^{\prime}\right\| \leq \frac{2}{2^{2^{k}-1}}\|x-\zeta\|=\frac{2}{2^{2^{k}-1}} \operatorname{dist}(x, V(f)),
$$

as wanted.

Our aim is to study the average behavior of the quantity $\gamma_{\text {worst }}(f)$. To this end, we must first consider some probability measure on $\mathcal{P}_{(d)}^{m}$. The following construction follows that of [SS93a, BCSS98].

For every positive integer number $l \in \mathbb{N}$, let $H_{l} \subseteq \mathbb{C}\left[X_{0}, \ldots, X_{n}\right]$ be the vector space of all homogenous polynomials of degree $l$ with coefficients in the field $\mathbb{C}$ of complex numbers. Let $\mathcal{H}_{(d)}^{m}:=\prod_{i=1}^{m} H_{d_{i}}$ be the complex vector space consisting of the polynomial systems of $m$ homogeneous polynomials $h:=\left[h_{1}, \ldots, h_{m}\right]$ of respective degrees $d_{i}$.

We denote by $\alpha$ a multi-index $\alpha:=\left(\alpha_{0}, \ldots, \alpha_{n}\right) \in \mathbb{Z}^{n+1}, \alpha_{i} \geq 0 \forall i$, and we denote $|\alpha|:=\alpha_{0}+\ldots+\alpha_{n}$. Then we write

$$
\underline{X}^{\alpha}:=X_{0}^{\alpha_{0}} \cdots X_{n}^{\alpha_{n}} .
$$

As in SS93a, BCSS98, Mal94, Dég01, we consider the Bombieri-Weyl Hermitian product in $H_{d_{i}}$, defined as follows. Fix $i, 1 \leq i \leq m$, and let $h, h^{\prime} \in H_{d_{i}}$ be two elements,

Then, we define

$$
h=\sum_{|\alpha|=d_{i}} a_{\alpha} \underline{X}^{\alpha}, \quad h^{\prime}=\sum_{|\alpha|=d_{i}} b_{\alpha} \underline{X}^{\alpha} .
$$

$$
\left\langle h, h^{\prime}\right\rangle_{\Delta_{i}}:=\sum_{|\alpha|=d_{i}}\left(\begin{array}{c}
d_{i} \\
\alpha
\end{array}\right)^{-1} a_{\alpha} \overline{b_{\alpha}},
$$

where $\overline{b_{\alpha}}$ is the complex conjugate of $b_{\alpha}$ and $\left(\begin{array}{c}d_{i} \\ \alpha\end{array}\right)$ is the multinomial coefficient. Namely,

$$
\left(\begin{array}{c}
d_{i} \\
\alpha
\end{array}\right)=\frac{d_{i} !}{\alpha_{0} ! \cdots \alpha_{n} !} \in \mathbb{N} .
$$

This Hermitian product induces an Hermitian product in $\mathcal{H}_{(d)}^{m}$ (which will also be called the Bombieri-Weyl Hermitian product) as follows. For any two elements $h:=\left[h_{1}, \ldots, h_{m}\right], h^{\prime}:=\left[h_{1}^{\prime}, \ldots, h_{m}^{\prime}\right]$ of $\mathcal{H}_{(d)}^{m}$, we define

$$
\left\langle h, h^{\prime}\right\rangle_{\Delta}:=\sum_{i=1}^{m}\left\langle h_{i}, h_{i}^{\prime}\right\rangle_{\Delta_{i}} .
$$

We consider the following mapping:

$$
\begin{aligned}
\Theta: \mathcal{P}_{(d)}^{m} & \longrightarrow \mathcal{H}_{(d)}^{m} \\
f & \mapsto \Theta(f),
\end{aligned}
$$

where $\Theta(f)$ is the homogenized counterpart of $f$. Namely, $\Theta(f)$ is obtained by adding a new unknown $X_{0}$ to homogenize all the monomials of each equation to the same degree $d_{i}$.

In this context, the solutions of $f$ are related to some of the solutions of $\Theta(f)$ as follows: If $\left(x_{0}, \ldots, x_{n}\right)$ is a solution of $\Theta(f)$, with $x_{0} \neq 0$, then

$$
\left(1, \frac{x_{1}}{x_{0}}, \ldots, \frac{x_{n}}{x_{0}}\right)
$$


is a solution of $f$. Conversely, if $\left(x_{1}, \ldots, x_{n}\right)$ is a solution of $f$, then

$$
\left(1, x_{1}, \ldots, x_{n}\right)
$$

is a solution of $\Theta(f)$.

The Hermitian product $\langle\cdot, \cdot\rangle_{\Delta}$ induces a Riemannian structure (and a metric) in the space $\mathcal{H}_{(d)}^{m}$. We define the Riemannian structure (and metric) in $\mathcal{P}_{(d)}^{m}$ to be the only one that makes $\Theta$ an isomorphism. We also denote $\|f\|_{\Delta}:=\|\Theta(f)\|_{\Delta}$.

Observe that the affine invariant $\gamma_{\text {worst }}(f)$ we have defined for $f \in \mathcal{P}_{(d)}^{m}$ does not vary if we multiply $f$ by a nonzero complex number. In other words, $\gamma_{\text {worst }}$ is a degree zero homogeneous function. Thus, the average behavior of $\gamma_{\text {worst }}$ in $\mathcal{P}_{(d)}^{m}$ can be calculated with Gaussian measure for the Bombieri-Weyl Hermitian product or, equivalently, in the sphere of radius 1 or the associated projective space $\mathbb{P}\left(\mathcal{P}_{(d)}^{m}\right)(\mathrm{cf}$. for example [BCSS98, page 208]). Namely, we are interested in the quantity

$$
\left.\mathrm{E}_{\mathbb{P}(\mathcal{P}}^{m}\right)\left[\gamma_{\text {worst }}\right] \equiv \mathrm{E}_{\mathcal{P}_{(d)}^{m}}\left[\gamma_{\text {worst }}\right]
$$

The isometry $\Theta$ also defines an isometry between the associated projective spaces $\mathbb{P}\left(\mathcal{P}_{(d)}^{m}\right)$ and $\mathbb{P}\left(\mathcal{H}_{(d)}^{m}\right)$. We will concentrate our efforts in the study of homogeneous projective systems $h \in \mathbb{P}\left(\mathcal{H}_{(d)}^{m}\right)$ and their set of projective solutions.

For a homogeneous polynomial system $h \in \mathcal{H}_{(d)}^{m}$, we denote by $V_{\mathbb{P}}(h) \subseteq \mathbb{P}_{n}(\mathbb{C})$ the set of projective solutions of $h$. Namely,

$$
V_{\mathbb{P}}(h):=\left\{\zeta \in \mathbb{P}_{n}(\mathbb{C}): h(\zeta)=0\right\} .
$$

Observe that for almost all systems $f \in \mathcal{P}_{(d)}^{m}$, the sets $V_{\mathbb{P}}(\Theta(f))$ and $V_{\mathbb{P}}(f)$ are projective varieties of dimension $n-m$. Also, we have that $V_{\mathbb{P}}(f) \subseteq V_{\mathbb{P}}(\Theta(f))$ (cf. Kun85]). Moreover, for almost all systems $f \in \mathcal{P}_{(d)}^{m}$, the following inequality also holds:

$$
\operatorname{dim}\left(V_{\mathbb{P}}(\Theta(f)) \cap\left\{X_{0}=0\right\}\right)=n-m-1 .
$$

Thus, except for a zero measure set in $\mathcal{P}_{(d)}^{m}$, the set $V_{\mathbb{P}}(\Theta(f)) \backslash V_{\mathbb{P}}(f)$ is contained in a projective variety of dimension at most $n-m-1$. We conclude that, for almost all $f \in \mathcal{P}_{(d)}^{m}$, the following equality holds:

$$
\nu\left[V_{\mathbb{P}}(f)\right]=\nu\left[V_{\mathbb{P}}(\Theta(f))\right] .
$$

In a similar way, for an integrable function $\psi: V_{\mathbb{P}}(\Theta(f)) \longrightarrow[0,+\infty]$, we have that

$$
\mathrm{E}_{V_{\mathbb{P}}(f)}[\psi]=\mathrm{E}_{V_{\mathbb{P}}(\Theta(f))}[\psi]
$$

for almost all $f \in \mathcal{P}_{(d)}^{m}$.

The main property of the Hermitian product $\langle\cdot, \cdot\rangle_{\Delta}$ defined above is its unitary invariance, which may be expressed as follows (cf. [BCSS98, pg. 218] and references therein). Let $h, h^{\prime} \in \mathcal{H}_{(d)}^{m}$. Let $U \in \mathcal{U}_{n+1}$ be a unitary matrix. Consider the elements $h \circ U, h^{\prime} \circ U \in \mathcal{H}_{(d)}^{m}$. Then, the following equality holds:

$$
\left\langle h \circ U, h^{\prime} \circ U\right\rangle_{\Delta}=\left\langle h, h^{\prime}\right\rangle_{\Delta} .
$$

The Riemannian structure of $\mathcal{H}_{(d)}^{m}$ induces a Riemannian structure in $\mathbb{P}\left(\mathcal{H}_{(d)}^{m}\right)$ in a natural way. Let $h \in \mathbb{P}\left(\mathcal{H}_{(d)}^{m}\right)$ be any element, and let

$$
h^{\perp}:=\left\{h^{\prime} \in \mathcal{H}_{(d)}^{m}:\left\langle h, h^{\prime}\right\rangle_{\Delta}=0\right\}
$$


be the orthogonal complement of $h$ in $\mathcal{H}_{(d)}^{m}$. The Hermitian product in $h^{\perp}$ is the one inherited from that of $\mathcal{H}_{(d)}^{m}$. Let $\bar{h}$ be any affine representation of $h$ such that $\|\bar{h}\|_{\Delta}=1$. Consider the affine chart

$$
\varphi_{\bar{h}}: h^{\perp} \longrightarrow \mathbb{P}\left(\mathcal{H}_{(d)}^{m}\right) \backslash h^{\perp},
$$

sending each point $h^{\prime} \in h^{\perp}$ to the projective class defined by $\bar{h}+h^{\prime}$. Then, $\varphi_{\bar{h}}$ is a diffeomorphism. Moreover, the tangent mapping $d_{0}\left(\varphi_{\bar{h}}\right)$ is a linear isometry. Thus, we may identify $T_{h} \mathbb{P}\left(\mathcal{H}_{(d)}^{m}\right)$ and $h^{\perp}$ via $\varphi_{\bar{h}}$. This Riemannian structure in $\mathbb{P}\left(\mathcal{H}_{(d)}^{m}\right)$ is unitarily invariant. Namely, for every unitary matrix $U \in \mathcal{U}_{n+1}$, the following mapping is an isometry:

$$
\begin{aligned}
\mathbb{P}\left(\mathcal{H}_{(d)}^{m}\right) & \longrightarrow \mathbb{P}\left(\mathcal{H}_{(d)}^{m}\right) \\
f & \mapsto f \circ U^{-1} .
\end{aligned}
$$

As for the space of solutions $\mathbb{P}_{n}(\mathbb{C})$, we consider it endowed with the usual Riemannian structure. For any point $x \in \mathbb{P}_{n}(\mathbb{C})$ and for any affine representation $\bar{x}$ of $x$, such that $\|\bar{x}\|_{2}=1$, we may identify $T_{x} \mathbb{P}_{n}(\mathbb{C}) \equiv x^{\perp}:=\left\{y \in \mathbb{C}^{n+1}:\langle x, y\rangle_{2}=\right.$ 0\} via the affine chart

$$
\begin{array}{cccc}
\varphi_{\bar{x}}: x^{\perp} & \longrightarrow \mathbb{P}_{n}(\mathbb{C}) \backslash x^{\perp} \\
y & \mapsto & \bar{x}+y .
\end{array}
$$

Observe that for any unitary matrix $U \in \mathcal{U}_{n+1}$, the following mapping is an isometry:

$$
\begin{array}{ccc}
\mathbb{P}_{n}(\mathbb{C}) & \longrightarrow & \mathbb{P}_{n}(\mathbb{C}) \\
x & \mapsto & U x .
\end{array}
$$

We will use the general notation $\nu[A]$ to denote the volume of the set $A$, where the dimension of $A$ is fixed by the context. For every positive integer $k \geq 0$, we denote by $\nu\left[\mathbb{P}_{k}(\mathbb{C})\right]$ the volume of the $k$-dimensional complex projective space. Namely,

$$
\nu\left[\mathbb{P}_{k}(\mathbb{C})\right]:=\frac{\pi^{k}}{\Gamma(k+1)}=\frac{\pi^{k}}{k !} .
$$

Note that the following equality also holds (cf. for example [BCSS98]):

$$
\nu\left[\mathbb{P}\left(\mathcal{H}_{(d)}^{m}\right)\right]=\frac{\pi^{N}}{\Gamma(N+1)} .
$$

For a linear space $\mathbb{C}^{k+1}$ and a positive real number $t>0$ we denote by $S^{t}\left(\mathbb{C}^{k+1}\right) \subseteq$ $\mathbb{C}^{k+1}$ the sphere of radius $t$ centered at 0 . Observe that the volume of $S^{1}\left(\mathbb{C}^{k+1}\right)$ as a submanifold of $\mathbb{C}^{k+1}$ is equal to

$$
2 \pi \nu\left[\mathbb{P}_{k}(\mathbb{C})\right]=\frac{2 \pi^{k+1}}{\Gamma(k+1)}
$$

Given any pair $(h, x) \in \mathbb{P}\left(\mathcal{H}_{(d)}^{m}\right) \times \mathbb{P}_{n}(\mathbb{C})$, we denote by $T_{x} h:=\left.\left(d_{x} h\right)\right|_{x^{\perp}}$ the restriction of the tangent mapping $d_{x} h$ to the tangent space $x^{\perp}$, where $h, x$ are any fixed affine representations such that $\|h\|_{\Delta}=\|x\|_{2}=1$. Sometimes we identify $T_{x} h$ and the Jacobian matrix in any orthonormal basis of $x^{\perp}$. In the case that $x=e_{0}:=(1: 0: \cdots: 0)$, we identify

$$
T_{e_{0}} h \equiv\left(\begin{array}{ccc}
\frac{\partial h_{1}}{\partial X_{1}}\left(e_{0}\right) & \cdots & \frac{\partial h_{1}}{\partial X_{n}}\left(e_{0}\right) \\
\vdots & & \vdots \\
\frac{\partial h_{m}}{\partial X_{1}}\left(e_{0}\right) & \cdots & \frac{\partial h_{m}}{\partial X_{n}}\left(e_{0}\right)
\end{array}\right),
$$

for any fixed representation $h \in \mathcal{H}_{(d)}^{m},\|h\|_{\Delta}=1$. 
2.2. The incidence variety. Let $W \subseteq \mathbb{P}\left(\mathcal{H}_{(d)}^{m}\right) \times \mathbb{P}_{n}(\mathbb{C})$ be the so-called incidence variety. Namely,

$$
W:=\left\{(h, \zeta) \in \mathbb{P}\left(\mathcal{H}_{(d)}^{m}\right) \times \mathbb{P}_{n}(\mathbb{C}): \zeta \in V_{\mathbb{P}}(h)\right\} .
$$

The result below is [BCSS98, Prop. 1, pg. 193].

Proposition 2.2. The incidence variety $W$ is a differentiable manifold of (complex) dimension $N+n-m$. Moreover, let $(h, \zeta) \in W$ be a point, and let $\bar{h}, \bar{\zeta}$ be affine representations of $h, \zeta$ such that $\|\bar{h}\|_{\Delta}=\|\bar{\zeta}\|_{2}=1$. Then, the tangent space $T_{(h, \zeta)} W \subseteq h^{\perp} \times \zeta^{\perp}$ can be identified with the space given by the following expression:

$$
T_{(h, \zeta)} W \equiv\left\{\left(h^{\prime}, x\right) \in h^{\perp} \times \zeta^{\perp}: h^{\prime}(\bar{\zeta})+\left(d_{\bar{\zeta}} \bar{h}\right) x=0\right\},
$$

where $d_{\bar{\zeta}} \bar{h}$ holds for the differential mapping of $\bar{h}$ at $\bar{\zeta}$. The identification with $T_{(h, \zeta)} W$ is given via the isometry

$$
d_{(0,0)}\left(\varphi_{\bar{h}} \times \varphi_{\bar{\zeta}}\right)
$$

As we have said above, for every unitary matrix $U \in \mathcal{U}_{n+1}, U$ defines isometries in $\mathbb{P}\left(\mathcal{H}_{(d)}^{m}\right)$ and $\mathbb{P}_{n}(\mathbb{C})$. Moreover, $U W=W$, and $U$ also defines an isometry in $W$. For every point $x \in \mathbb{P}_{n}(\mathbb{C})$ we denote by $V_{x}$ the linear subspace of $\mathbb{P}\left(\mathcal{H}_{(d)}^{m}\right)$ given as

$$
V_{x}:=\left\{h \in \mathbb{P}\left(\mathcal{H}_{(d)}^{m}\right): x \in V_{\mathbb{P}}(h)\right\} .
$$

We consider the two canonical projections

$$
p_{1}: W \longrightarrow \mathbb{P}\left(\mathcal{H}_{(d)}^{m}\right), \quad p_{2}: W \longrightarrow \mathbb{P}_{n}(\mathbb{C}) .
$$

We can obviously identify $p_{1}^{-1}(h)$ and $V_{\mathbb{P}}(h)$. In the same way, we can identify $p_{2}^{-1}(x)$ and $V_{x}$. From now on, we do not distinguish between those concepts.

2.3. The condition number of linear systems. Condition numbers in Linear Algebra were introduced by A. Turing in Tur48. They were also studied by J. von Neumann and collaborators (cf. [NG47]) and by J.H. Wilkinson (cf. also [Wil65]). Variations of these condition numbers may be found in the literature of Numerical Linear Algebra (cf. Dem88, GVL96], Hig02, TB97] and the references therein).

We will denote by $\kappa_{D}^{m}$ the generalized Condition Number of Linear Algebra (cf. for example [SS90, BP05]). Namely, let $k \geq m$ be two positive integers. Then, for a rank $m$ matrix $A \in \mathcal{M}_{m \times k}(\mathbb{C})$,

$$
\kappa_{D}^{m}(A):=\|A\|_{F}\left\|A^{\dagger}\right\|_{2},
$$

where $\|\cdot\|_{F}$ is the Frobenius norm and $A^{\dagger}$ holds for the Moore-Penrose inverse of $A$. It is well known (cf. [SS90, BP05, Kah00]) that the condition number $\kappa_{D}^{m}$ controls the stability of the kernel or the Moore-Penrose inverse calculations. Moreover, some bounds on the probability distribution of $\kappa_{D}^{m}$ have been obtained since [BP06a, BP05] appeared. Namely, we have the following result.

Lemma 2.3. Let $n \geq m \geq 2$ be two positive integers. For any positive real number $s>0$, the following inequality holds:

$$
\frac{\nu\left[\left\{M \in \mathbb{P}\left(\mathcal{M}_{m \times(n+1)}(\mathbb{C})\right): \kappa_{D}^{m}(M)>s^{-1}\right\}\right]}{\nu\left[\mathbb{P}\left(\mathcal{M}_{m \times(n+1)}(\mathbb{C})\right)\right]} \leq 2\left(\frac{e m^{3 / 2}(n+1)}{n-m+2} s\right)^{2(n-m+2)} .
$$


Now, let $m=1$. Then, the following equality holds:

$$
\frac{\left.\nu\left\{M \in \mathbb{P}\left(\mathcal{M}_{1 \times(n+1)}(\mathbb{C})\right): \kappa_{D}^{1}(M)>s^{-1}\right\}\right]}{\nu\left[\mathbb{P}_{n}(\mathbb{C})\right]}=\left\{\begin{array}{l}
1 \text { if } s>1, \\
0 \text { if } s \leq 1 .
\end{array}\right.
$$

Proof. The first part of the lemma is from [BP06a]. As for the second part, observe that for every nonzero matrix $A \in \mathcal{M}_{1 \times(n+1)}(\mathbb{C})$, the following equality holds:

$$
\kappa_{D}^{1}(A)=1 \text {. }
$$

The upper bound on the probability distribution of $\kappa_{D}^{m}$ may be translated into a bound on the expected value $\mathrm{E}_{\mathbb{P}\left(\mathcal{M}_{m \times(n+1)}(\mathbb{C})\right)}\left[\kappa_{D}^{m}\right]$, using the following result.

Lemma 2.4. Let $X$ be a positive real-valued random variable such that for every positive real number $t>1$,

$$
\operatorname{Prob}[X>t] \leq c t^{-\alpha},
$$

where Prob[·] holds for Probability, and $c>1, \alpha>1$ are some positive constants. Then, the following inequality holds:

$$
\mathrm{E}[X] \leq c^{\frac{1}{\alpha}} \frac{\alpha}{\alpha-1} .
$$

Proof. We use the following equality, which is a well-known fact from Probability Theory.

$$
\mathrm{E}[X]=\int_{0}^{\infty} \operatorname{Prob}[X>t] d t .
$$

Then, observe that for every positive real number $s>1$,

$$
\mathrm{E}[X]=\int_{0}^{\infty} \operatorname{Prob}[X>t] d t \leq s+c \int_{s}^{\infty} t^{-\alpha} d t=s+c \frac{s^{1-\alpha}}{\alpha-1} .
$$

Let $s:=c^{\frac{1}{\alpha}}$, and the lemma follows.

Corollary 2.5. Let $n \geq m \geq 2$ be two positive integers. Then, the expected value of $\kappa_{D}^{m}$ satisfies:

$$
\mathrm{E}_{\mathbb{P}\left(\mathcal{M}_{m \times(n+1)}(\mathbb{C})\right)}\left[\kappa_{D}^{m}\right] \leq \frac{2^{1 / 4} e m^{3 / 2}(n+1)}{n-m+3 / 2} .
$$

Now, let $n \geq m=1$. Then, we have that

$$
\mathrm{E}_{\mathbb{P}\left(\mathcal{M}_{1 \times(n+1)}(\mathbb{C})\right)}\left[\kappa_{D}^{1}\right]=1 .
$$

Proof. The inequality follows directly from Lemmas 2.3 and 2.4. The equality is due to the fact that $\kappa_{D}^{1}(M)=1$ for every nonzero matrix $M \in \mathcal{M}_{1 \times(n+1)}(\mathbb{C})$.

2.4. The condition number of nonlinear systems. In the series of papers SS93a, SS93b, SS93c, SS94, SS96] a condition number for nonlinear zero-dimensional systems of equations is proposed and analyzed. In Dég01, an extension of this condition number for the underdetermined case is suggested, and some interesting properties are shown. The projective version of this condition number may be defined as follows: 
Let $h \in \mathbb{P}\left(\mathcal{H}_{(d)}^{m}\right)$, and let $\zeta \in V_{\mathbb{P}}(h)$ be a regular solution of $h$. We also denote by $h$ and $\zeta$ any respective affine representations of these projective points. Then, the condition number $\mu_{\text {norm }}^{m}(h, \zeta)$ is defined as follows:

$$
\mu_{\text {norm }}^{m}(h, \zeta):=\|h\|_{\Delta}\left\|\left(\left.d_{\zeta} h\right|_{\zeta^{\perp}}\right)^{\dagger} \operatorname{Diag}\left(\|\zeta\|^{d_{i}-1} d_{i}^{1 / 2}\right)\right\|_{2},
$$

where $\operatorname{Diag}\left(\|\zeta\|^{d_{i}-1} d_{i}^{1 / 2}\right):=\operatorname{Diag}\left(d_{1}^{1 / 2}\|\zeta\|^{d_{1}-1}, \ldots, d_{m}^{1 / 2}\|\zeta\|^{d_{m}-1}\right)$ is this diagonal matrix. In the case that $\zeta$ is a singular solution of $h$ (i.e., the differential mapping $d_{\zeta} h$ is not surjective) we define $\mu_{\text {norm }}^{m}(h, \zeta):=+\infty$. Note that the following equality holds:

$$
\mu_{\text {norm }}^{m}(h, \zeta)=\frac{\kappa_{D}^{m}\left(\operatorname{Diag}\left(d_{i}^{-1 / 2}\right) T_{\zeta} h\right)}{\left\|\operatorname{Diag}\left(d_{i}^{-1 / 2}\right) T_{\zeta} h\right\|_{F}},
$$

where $\operatorname{Diag}\left(d_{i}^{-1 / 2}\right):=\operatorname{Diag}\left(d_{1}^{-1 / 2}, \ldots, d_{m}^{-1 / 2}\right)$ is this diagonal matrix, and $T_{\zeta} h$ is as defined in Section 2.1 .

The quantity $\mu_{\text {norm }}^{m}$ depends both on the system and the solution. Then, we consider two possible definitions for the condition number of a polynomial system $h \in \mathbb{P}\left(\mathcal{H}_{(d)}^{m}\right):$

$$
\mu_{\mathrm{worst}}^{m}(h):=\max _{\zeta \in V_{\mathbb{P}}(h)} \mu_{\mathrm{norm}}^{m}(h, \zeta), \quad \mu_{\mathrm{av}}^{m}(h):=\mathrm{E}_{V_{\mathbb{P}}(h)}\left[\mu_{\mathrm{norm}}^{m}(h, \cdot)\right] .
$$

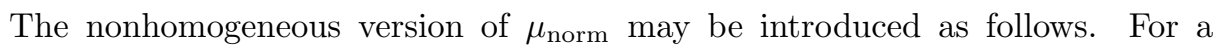
polynomial $f \in \mathcal{P}_{(d)}^{m}$ and a solution $\zeta \in V(f)$, we define

$$
\mu_{\text {norm }}^{m}(f, \zeta):=\mu_{\text {norm }}^{m}(\Theta(f),(1, \zeta)),
$$

where $\Theta$ is the mapping of Section 2.1 (note that $(1, \zeta) \in V_{\mathbb{P}}(\Theta(f))$ ). The nonhomogeneous versions of $\mu_{\text {worst }}$ and $\mu_{\mathrm{av}}$ have been defined in the Introduction (see identities (1.3), (1.4)).

Observe that, as $d_{\zeta} f$ varies in a continuous fashion with $\zeta$, for every $f \in \mathcal{P}_{(d)}^{m}$ we have

$$
\begin{aligned}
\mu_{\text {worst }}^{m}(f) & =\sup _{\zeta \in V(f)} \mu_{\text {norm }}^{m}(\Theta(f),(1, \zeta))=\max _{\zeta \in V_{\mathbb{P}}(f)} \mu_{\text {norm }}^{m}(\Theta(f),(1, \zeta)) \\
& \leq \max _{\zeta \in V_{\mathbb{P}}(\Theta(f))} \mu_{\text {norm }}^{m}(\Theta(f), \zeta)=\mu_{\text {worst }}^{m}(\Theta(f))
\end{aligned}
$$

From the definitions and equation (2.1) above, the following equality holds for almost all $f \in \mathcal{P}_{(d)}^{m}$ :

$$
\mu_{\mathrm{av}}^{m}(f)=\mu_{\mathrm{av}}^{m}(\Theta(f)) .
$$

2.5. Some geometric integration theory. We will make extensive use of the so-called Coarea Formula, a classical integral formula which generalizes Fubini's Theorem. The most general version we know is Federer's Coarea Formula (cf. [Fed69]), but for our purposes a smooth version as used in BCSS98 or SS93b] suffices.

Definition 2.6. Let $X$ and $Y$ be Riemannian manifolds, and let $F: X \longrightarrow Y$ be a $C^{1}$ surjective map. Let $k:=\operatorname{dim}(Y)$ be the real dimension of $Y$. For every point $x \in X$ such that $d_{x} F$ is surjective, let $v_{1}^{x}, \ldots, v_{k}^{x}$ be an orthonormal basis of $\operatorname{Ker}\left(d_{x} F\right)^{\perp}$. Then, we define the Normal Jacobian of $F$ at $x, N J_{x} F$, as the volume in $T_{F(x)} Y$ of the parallelepiped spanned by $d_{x} F\left(v_{1}^{x}\right), \ldots, d_{x} F\left(v_{k}^{x}\right)$. In the case that $d_{x} F$ is not surjective, we define $N J_{x} F:=0$. 
Theorem 2.7 (Coarea Formula). Let $X, Y$ be two Riemannian manifolds of respective dimensions $k_{1} \geq k_{2}$. Let $F: X \longrightarrow Y$ be a $C^{1}$ surjective map such that the differential mapping $d_{x} F$ is surjective for almost all $x \in X$. Let $\psi: X \longrightarrow \mathbb{R}$ be an integrable mapping. Then, the following equality holds:

$$
\int_{X} \psi d X=\int_{y \in Y} \int_{x \in F^{-1}(y)} \psi(x) \frac{1}{N J_{x} F} d\left(F^{-1}(y)\right) d Y,
$$

where $N J_{x} F$ is the normal Jacobian of $F$ at $x$.

Observe that the integral on the right-hand side of equation (2.5) may be interpreted as follows: From Sard's Theorem, for every $y \in Y$ except for a zero measure set, $y$ is a regular value of $F$. Hence, $F^{-1}(y)$ is a differentiable manifold of dimension $k_{1}-k_{2}$, and it inherits from $X$ a structure of a Riemannian manifold. Thus, it makes sense to integrate functions on $F^{-1}(y)$.

The following proposition immediately follows from the definition (see for example [BCSS98, pg. 244] or Bel06, Cor. 1.1.12]).

Proposition 2.8. Let $X, Y$ be two Riemannian manifolds, and let $F: X \longrightarrow Y$ be a $C^{1}$ map. Let $x_{1}, x_{2} \in X$ be two points. Assume that there exist isometries $\varphi_{X}: X \longrightarrow X$ and $\varphi_{Y}: Y \longrightarrow Y$ such that $\varphi_{X}\left(x_{1}\right)=x_{2}$, and

$$
F \circ \varphi_{X}=\varphi_{Y} \circ F \text {. }
$$

Then, the following equality holds:

$$
N J_{x_{1}} F=N J_{x_{2}} F .
$$

Moreover, if there exists an inverse $G: Y \longrightarrow X$, then

$$
N J_{x} F=\frac{1}{N J_{F(x)} G} \text {. }
$$

\section{Condition number And CONVERGEnCE RADius}

In Section 2.1 we have introduced the quantity $\gamma_{\text {worst }}$ to control the convergence of Newton iterations. The quantity $\gamma_{\text {worst }}$ is defined in $\mathcal{P}_{(d)}^{m}$ (or in the associated projective space). Later, in Section 2.4 we have centered our attention on the condition number $\mu_{\text {norm }}^{m}$. This condition number $\mu_{\text {norm }}^{m}$ has been defined in the projective space $\mathbb{P}\left(\mathcal{H}_{(d)}^{m}\right)$ (or, equivalently, in the affine space $\mathcal{H}_{(d)}^{m}$ ), and also in the space $\mathcal{P}_{(d)}^{m}$. Now we will relate these concepts. We start with the following elementary lemma.

Lemma 3.1. Let $h \in \mathcal{H}_{(d)}^{m}, \zeta \in \mathbb{C}^{n+1}$ be such that $h(\zeta)=0, \operatorname{rank}\left(T_{\zeta} h\right)=m$. Then, for every vector $v \in \mathbb{C}^{m}$, the following equality holds:

$$
\left(d_{\zeta} h\right)^{\dagger} v=\left(\left.\left(d_{\zeta} h\right)\right|_{\zeta^{\perp}}\right)^{\dagger} v .
$$

Proof. For an onto linear operator between Hilbert spaces $L: E_{1} \longrightarrow E_{2}$, we have that

$$
L^{\dagger}=i \circ\left(L_{(\operatorname{Ker} L)^{\perp}}\right)^{-1},
$$

where $i$ is the inclusion in $E_{1}$. Now, observe that $h \in \mathcal{H}_{(d)}^{m}$ is a system of homogeneous polynomials and $\zeta \in V_{\mathbb{P}}(h)$ is a solution of $h$. Hence $d_{\zeta} h(\zeta)=0$. Thus,

$$
\left(d_{\zeta} h\right)^{\dagger}=i \circ\left(\left.\left(d_{\zeta} h\right)\right|_{\left(\operatorname{Ker}\left(d_{\zeta} h\right)\right)^{\perp}}\right)^{-1},
$$


and

$$
\left(\left.\left(d_{\zeta} h\right)\right|_{\zeta^{\perp}}\right)^{\dagger}=i_{*} \circ\left(\left.\left(d_{\zeta} h\right)\right|_{\left(\operatorname{Ker}\left(d_{\zeta} h\right)\right)^{\perp}}\right)^{-1},
$$

where $i$ is the inclusion in $\mathbb{C}^{n+1}$ and $i_{*}$ is the inclusion in $\zeta^{\perp}$. The lemma follows.

We now define a projective version of the quantity $\gamma$. Let $(h, \zeta) \in W$ be a point in the incidence variety, such that $d_{\zeta} h$ is surjective. Then, we define

$$
\gamma_{0}(h, \zeta):=\|\zeta\|_{2} \sup _{k \geq 2}\left\|\left(\left.\left(d_{\zeta} h\right)\right|_{\zeta^{\perp}}\right)^{\dagger} \frac{d_{\zeta}^{(k)} h}{k !}\right\|_{2}^{1 /(k-1)} .
$$

In the case that $d_{\zeta} h$ is not surjective, we define $\gamma_{0}(h, \zeta):=+\infty$. This definition is independent of the representatives of $h$ and $\zeta$ used in the formula. Observe that $\gamma_{0}$ is only defined for homogeneous systems, while $\gamma$ (as defined in Section 2.1) is also defined for nonhomogeneous systems. Finally, another quantity will help us to prove our main theorems. The following is a nonhomogeneous version of the condition number $\mu_{\text {norm }}^{m}$. For $f \in \mathcal{P}_{(d)}^{m}$ and $\zeta \in V(f)$, we define

$$
\mu_{\text {affine }}^{m}(f, \zeta):=\|f\|_{\Delta}\left\|\left(d_{\zeta} f\right)^{\dagger} \operatorname{Diag}\left(d_{i}^{1 / 2}\|(1, \zeta)\|^{d_{i}-1}\right)\right\|_{2} .
$$

Note that $\mu_{\text {affine }}^{m}(f, \zeta)$ is not equal to $\mu_{\text {norm }}^{m}(f, \zeta)$ in general. The quantity $\mu_{\text {affine }}^{m}$ will only be used in intermediate results. All these concepts will be related in subsequent lemmas.

The following result is easily proved following the arguments in BCSS98, Sect. 14.2] or [SS93a]. It relates $\gamma_{0}$ with $\mu_{\text {norm }}^{m}$ and $\gamma$ with $\mu_{\text {affine }}^{m}$.

Lemma 3.2. Let $(h, \zeta) \in W$ be a point in the incidence variety. Then, the following inequality holds:

$$
\gamma_{0}(h, \zeta) \leq \frac{d^{3 / 2}}{2} \mu_{\text {norm }}^{m}(h, \zeta)
$$

Moreover, let $f \in \mathcal{P}_{(d)}^{m}$ and let $\zeta \in V(f) \subseteq \mathbb{C}^{n}$ be a solution of $f$. Then,

$$
\|(1, \zeta)\|_{2} \gamma(f, \zeta) \leq \frac{d^{3 / 2}}{2} \mu_{\text {affine }}^{m}(f, \zeta) .
$$

Proof. It suffices to prove the result in the case that $\zeta$ is a regular solution of $h$ (resp. $f$ ). We start with the projective case. We consider fixed some representatives of $h$, $\zeta$. Let $h=\left[h_{1}, \ldots, h_{m}\right]$ be given by the list of its polynomials. From the definition, for every $k>1$,

$$
\begin{gathered}
\left(\frac{\left\|\operatorname{Diag}\left(\|\zeta\|^{k-d_{i}} d_{i}^{-1 / 2}\right) d_{\zeta}^{(k)} h\right\|_{2}}{\|h\|_{\Delta} k !}\right)^{1 /(k-1)} \leq\left(\sum_{i=1}^{m}\left(\frac{\left\|d_{\zeta}^{(k)} h_{i}\right\|_{2}}{\|\zeta\|_{2}^{d_{i}-k}\|h\|_{\Delta} d_{i}^{1 / 2} k !}\right)^{2}\right)^{1 / 2(k-1)} \\
=\left(\sum_{i=1}^{m}\left(\frac{\left\|d_{\zeta}^{(k)} h_{i}\right\|_{2}}{\|\zeta\|_{2}^{d_{i}-k}\left\|h_{i}\right\|_{\Delta_{i}} d_{i}^{1 / 2} k !} \frac{\left\|h_{i}\right\|_{\Delta_{i}}}{\|h\|_{\Delta}}\right)^{2}\right)^{1 / 2(k-1)} .
\end{gathered}
$$

From [BCSS98, Lem. 11, pg. 269], this last is at most

$$
\left(\sum_{i=1}^{m}\left(\left(\frac{d_{i}^{3 / 2}}{2}\right)^{k-1} \frac{\left\|h_{i}\right\|_{\Delta_{i}}}{\|h\|_{\Delta}}\right)^{2}\right)^{1 / 2(k-1)} \leq \frac{d^{3 / 2}}{2\|h\|_{\Delta}}\left(\sum_{i=1}^{m}\left\|h_{i}\right\|_{\Delta_{i}}^{2}\right)^{1 / 2}=\frac{d^{3 / 2}}{2} .
$$


We have proved that for every $k>1$ and every $h \in \mathcal{H}_{(d)}^{m}, \zeta \in \mathbb{C}^{n+1}$, the following holds:

$$
\left(\frac{\left\|\operatorname{Diag}\left(\|\zeta\|^{k-d_{i}} d_{i}^{-1 / 2}\right) d_{\zeta}^{(k)} h\right\|_{2}}{\|h\|_{\Delta} k !}\right)^{1 /(k-1)} \leq \frac{d^{3 / 2}}{2} .
$$

Now, assume that we choose representatives such that $\|h\|_{\Delta}=\|\zeta\|_{2}=1$. Then, we can write

$$
\begin{gathered}
\gamma_{0}(h, \zeta)=\sup _{k \geq 2}\left\|\left(T_{\zeta} h\right)^{\dagger} \operatorname{Diag}\left(d_{i}^{1 / 2}\right) \operatorname{Diag}\left(d_{i}^{-1 / 2}\right) \frac{d_{\zeta}^{(k)} h}{k !}\right\|_{2}^{1 /(k-1)} \\
\leq \sup _{k \geq 2}\left\|\left(T_{\zeta} h\right)^{\dagger} \operatorname{Diag}\left(d_{i}^{1 / 2}\right)\right\|_{2}^{1 /(k-1)}\left(\frac{\left\|\operatorname{Diag}\left(d_{i}^{-1 / 2}\right) d_{\zeta}^{(k)} h\right\|_{2}}{k !}\right)^{1 /(k-1)} .
\end{gathered}
$$

From inequality (3.1), we obtain that

$$
\begin{aligned}
& \gamma_{0}(f, \zeta) \leq \frac{d^{3 / 2}}{2} \sup _{k \geq 2}\left\|\left(T_{\zeta} h\right)^{\dagger} \operatorname{Diag}\left(d_{i}^{1 / 2}\right)\right\|_{2}^{1 /(k-1)} \\
= & \frac{d^{3 / 2}}{2}\left\|\left(T_{\zeta} h\right)^{\dagger} \operatorname{Diag}\left(d_{i}^{1 / 2}\right)\right\|_{2}=\frac{d^{3 / 2}}{2} \mu_{\text {norm }}^{m}(h, \zeta),
\end{aligned}
$$

as wanted.

Finally, for the affine case, observe that

$$
\begin{gathered}
\|(1, \zeta)\|_{2} \gamma(f, \zeta)=\|(1, \zeta)\|_{2} \sup _{k \geq 2}\left\|\left(d_{\zeta} f\right)^{\dagger} \frac{d_{\zeta}^{(k)} f}{k !}\right\|_{2}^{\frac{1}{k-1}} \\
=\sup _{k \geq 2}\left\|\left(d_{\zeta} f\right)^{\dagger} \operatorname{Diag}\left(d_{i}^{1 / 2}\|(1, \zeta)\|^{d_{i}-1}\right) \operatorname{Diag}\left(d_{i}^{-1 / 2}\|(1, \zeta)\|^{k-d_{i}}\right) \frac{d_{\zeta}^{(k)} f}{k !}\right\|_{2}^{\frac{1}{k-1}} \\
\leq \sup _{k \geq 2} \mu_{\text {affine }}^{m}(f, \zeta)^{\frac{1}{k-1}} \sup _{k \geq 2}\left\|\operatorname{Diag}\left(\|(1, \zeta)\|^{k-d_{i}} d_{i}^{-1 / 2}\right) \frac{d_{\zeta}^{(k)} f}{\|f\|_{\Delta} k !}\right\|_{2}^{\frac{1}{k-1}} .
\end{gathered}
$$

Recall that for $f \in \mathcal{P}_{(d)}^{m}$, we have defined $\Theta(f) \in \mathcal{H}_{(d)}^{m}$ as the homogenized counterpart of $f$ (see Section 2.1). Then, observe that

$$
f=\left.\Theta(f)\right|_{\{1\} \times \mathbb{C}^{n}} .
$$

Hence, we have that

$$
\begin{gathered}
\sup _{k \geq 2}\left\|\operatorname{Diag}\left(\|(1, \zeta)\|^{k-d_{i}} d_{i}^{-1 / 2}\right) \frac{d_{\zeta}^{(k)} f}{\|f\|_{\Delta} k !}\right\|_{2}^{\frac{1}{k-1}} \\
\leq \sup _{k \geq 2}\left\|\operatorname{Diag}\left(\|(1, \zeta)\|^{k-d_{i}} d_{i}^{-1 / 2}\right) \frac{d_{\zeta}^{(k)} \Theta(f)}{\|\Theta(f)\|_{\Delta} k !}\right\|_{2}^{\frac{1}{k-1}} .
\end{gathered}
$$

As $\Theta(f) \in \mathcal{H}_{(d)}^{m}$ is a homogeneous polynomial and $(1, \zeta) \in \mathbb{C}^{n+1}$ is a solution of $\Theta(f)$, from inequality (3.1) we conclude that this last quantity is at most

$$
\frac{d^{3 / 2}}{2}
$$


and the lemma follows.

The result below relates the condition number $\mu_{\text {norm }}^{m}$ with its affine counterpart $\mu_{\text {affine }}^{m}$.

Lemma 3.3. Let $f \in \mathcal{P}_{(d)}^{m}$ be a system, $\zeta \in \mathbb{C}^{n}$ be a solution of $f$. Then, the following inequality holds:

$$
\mu_{\text {affine }}^{m}(f, \zeta) \leq\|(1, \zeta)\|_{2} \mu_{\text {norm }}^{m}(\Theta(f),(1, \zeta))=\|(1, \zeta)\|_{2} \mu_{\text {norm }}^{m}(f, \zeta) .
$$

Proof. Again, it suffices to check the case that $\zeta$ is a regular solution of $f$, which implies that $(1, \zeta)$ is a regular solution of $\Theta(f)$. Observe that $f=\left.\Theta(f)\right|_{\{1\} \times \mathbb{C}^{n}}$. Moreover, we have defined

$$
\|f\|_{\Delta}:=\|\Theta(f)\|_{\Delta}
$$

Hence, we can write

$$
\mu_{\text {affine }}^{m}(f, \zeta)=\|\Theta(f)\|_{\Delta}\left\|\left(\left.d_{(1, \zeta)} \Theta(f)\right|_{e_{0}^{\perp}}\right)^{\dagger} \operatorname{Diag}\left(d_{i}^{1 / 2}\|(1, \zeta)\|^{d_{i}-1}\right)\right\|_{2} .
$$

Now, observe that

$$
\begin{gathered}
\left\|\left(\left.d_{(1, \zeta)} \Theta(f)\right|_{e_{0}^{\perp}}\right)^{\dagger} \operatorname{Diag}\left(d_{i}^{1 / 2}\|(1, \zeta)\|^{d_{i}-1}\right)\right\|_{2}= \\
\left\|\left(\left.d_{(1, \zeta)} \Theta(f)\right|_{e_{0}^{\perp}}\right)^{\dagger}\left(\left.d_{(1, \zeta)} \Theta(f)\right|_{(1, \zeta)^{\perp}}\right)\left(\left.d_{(1, \zeta)} \Theta(f)\right|_{(1, \zeta)^{\perp}}\right)^{\dagger} \operatorname{Diag}\left(d_{i}^{1 / 2}\|(1, \zeta)\|^{d_{i}-1}\right)\right\|_{2} \leq \\
\left\|\left(\left.d_{(1, \zeta)} \Theta(f)\right|_{e_{0}^{\perp}}\right)^{\dagger}\left(\left.d_{(1, \zeta)} \Theta(f)\right|_{(1, \zeta)^{\perp}}\right)\right\|_{2}\left\|\left(\left.d_{(1, \zeta)} \Theta(f)\right|_{(1, \zeta)^{\perp}}\right)^{\dagger} \operatorname{Diag}\left(d_{i}^{1 / 2}\|(1, \zeta)\|^{d_{i}-1}\right)\right\|_{2} .
\end{gathered}
$$

From the definition of $\mu_{\text {norm }}^{m}$ we conclude:

$$
\mu_{\text {affine }}^{m}(f, \zeta) \leq \mu_{\text {norm }}^{m}(\Theta(f),(1, \zeta))\left\|\left(\left.d_{(1, \zeta)} \Theta(f)\right|_{e^{\perp}}\right)^{\dagger}\left(\left.d_{(1, \zeta)} \Theta(f)\right|_{(1, \zeta) \perp}\right)\right\|_{2} .
$$

Hence, it suffices to prove that for a homogeneous system $h \in \mathcal{H}_{(d)}^{m}$ and a solution $(1, \zeta)$ of $h$,

$$
\left\|\left(\left.d_{(1, \zeta)} h\right|_{e_{0}^{\perp}}\right)^{\dagger}\left(\left.d_{(1, \zeta)} h\right|_{(1, \zeta)^{\perp}}\right)\right\|_{2} \leq\|(1, \zeta)\|_{2} .
$$

We check this last inequality. In fact, let $w \in(1, \zeta)^{\perp}$ be a vector. If $w \in e_{0}^{\perp}$, then

$$
\begin{gathered}
\left\|\left(\left.d_{(1, \zeta)} h\right|_{e_{0}^{\perp}}\right)^{\dagger}\left(\left.d_{(1, \zeta)} h\right|_{(1, \zeta)^{\perp}}\right)(v)\right\|_{2} \\
=\left\|\left(\left.d_{(1, \zeta)} h\right|_{e_{0}^{\perp}}\right)^{\dagger}\left(\left.d_{(1, \zeta)} h\right|_{e_{0}^{\perp}}\right)(v)\right\|_{2} \leq\|v\|_{2},
\end{gathered}
$$

from elementary properties of the Moore-Penrose inverse (see for example [Ded06]). Assume now that $v \in(1, \zeta)^{\perp} \cap\left((1, \zeta)^{\perp} \cap e_{0}^{\perp}\right)^{\perp}$, which is a complex subspace of dimension 1. Then, $v=t\left(-\|\zeta\|_{2}^{2}, \zeta\right) \in \mathbb{C}^{n+1}$ for some $t \in \mathbb{C}$. Moreover, let $v$ be given by

$$
v=w-t\|\zeta\|_{2}^{2}(1, \zeta), \quad w \in e_{0}^{\perp} .
$$

Then,

$$
\begin{gathered}
\frac{1}{\|v\|_{2}}\left\|\left(\left.d_{(1, \zeta)} h\right|_{e_{0}^{\perp}}\right)^{\dagger}\left(\left.d_{(1, \zeta)} h\right|_{(1, \zeta)^{\perp}}\right)(v)\right\|_{2} \\
=\frac{1}{\|v\|_{2}}\left\|\left(\left.d_{(1, \zeta)} h\right|_{e_{0}^{\perp}}\right)^{\dagger}\left(d_{(1, \zeta)} h\right)(v)\right\|_{2} \\
=\frac{1}{\|v\|_{2}}\left\|\left(\left.d_{(1, \zeta)} h\right|_{e_{0}^{\perp}}\right)^{\dagger}\left(d_{(1, \zeta)} h\right)(w)\right\|_{2} \leq \frac{\|w\|_{2}}{\|v\|_{2}} .
\end{gathered}
$$

But

$$
\frac{\|w\|_{2}}{\|v\|_{2}}=\frac{\left\|t\left(-\|\zeta\|_{2}^{2}, \zeta\right)+t\right\| \zeta\left\|_{2}^{2}(1, \zeta)\right\|_{2}}{\left\|t\left(-\|\zeta\|_{2}^{2}, \zeta\right)\right\|_{2}}=\|(1, \zeta)\|_{2}
$$


This finishes the proof of the lemma.

Finally, we can relate the quantity $\gamma$ of Section 2.1 to the condition number $\mu_{\mathrm{norm}}^{m}$ of Section 2.4 as follows.

Proposition 3.4. Let $f \in \mathcal{P}_{(d)}^{m}$ be a system of polynomial equations, and let $\zeta$ be a solution of $f$. Then, the following inequality holds:

$$
\gamma(f, \zeta) \leq \frac{d^{3 / 2}}{2} \mu_{\text {norm }}^{m}(\Theta(f),(1, \zeta)) .
$$

Moreover, the following chain of inequalities also holds:

$$
\gamma_{\text {worst }}(f) \leq \frac{d^{3 / 2}}{2} \mu_{\text {worst }}^{m}(f) \leq \frac{d^{3 / 2}}{2} \mu_{\text {worst }}^{m}(\Theta(f)) .
$$

Proof. The second assertion immediately follows from the first one. From Lemma 3.2 ,

$$
\gamma(f, \zeta) \leq \frac{1}{\|(1, \zeta)\|_{2}} \frac{d^{3 / 2}}{2} \mu_{\text {affine }}^{m}(f, \zeta)
$$

From Lemma 3.3. this last quantity is at most

$$
\frac{1}{\|(1, \zeta)\|_{2}} \frac{d^{3 / 2}}{2}\|(1, \zeta)\|_{2} \mu_{\mathrm{norm}}^{m}(\Theta(f),(1, \zeta))
$$

as wanted.

From Proposition 3.4, we can reduce the problem of the average value of $\gamma_{\text {worst }}(f)$ in $\mathcal{P}_{(d)}^{m}$ to the study of the quantity

$$
\mathrm{E}_{\mathbb{P}\left(\mathcal{H}_{(d)}^{m}\right)}\left[\mu_{\mathrm{worst}}^{m}\right]
$$

Hence, we are interested in the integration of functions in the projective space of homogeneous polynomials $\mathbb{P}\left(\mathcal{H}_{(d)}^{m}\right)$. In the following sections we will face this problem, from a more general point of view.

\section{INTEGRATION ON THE SPACE OF POLYNOMIAL SYSTEMS}

In this section we follow the demonstration scheme of SS93b] to relate integration on the space of polynomial systems to integration on the space of linear systems. Namely, we obtain the following technical result.

Theorem 4.1. Let $(d)=\left(d_{1}, \ldots, d_{m}\right)$ be such that $d_{i}>1$ for some $i, 1 \leq i \leq m$. Let $\Phi:[0,+\infty] \longrightarrow[0,+\infty]$ be an integrable mapping. Let $\mathcal{J}_{\mathbb{P}}(\Phi)$ be the integral defined as follows:

$$
\mathcal{J}_{\mathbb{P}}(\Phi):=\int_{h \in \mathbb{P}\left(\mathcal{H}_{(d)}^{m}\right)} \int_{\zeta \in V_{\mathbb{P}}(h)} \Phi\left(\mu_{\text {norm }}^{m}(h, \zeta)\right) d V_{\mathbb{P}}(f) d \mathbb{P}\left(\mathcal{H}_{(d)}^{m}\right) .
$$

Moreover, for every real number $t \in[0,1]$, consider the following integral:

$$
\mathcal{I}_{\mathcal{M}}(\Phi, t):=\int_{M \in \mathbb{P}\left(\mathcal{M}_{m \times(n+1)}(\mathbb{C})\right)} \Phi\left(\frac{\kappa_{D}^{m}(M)}{t}\right) d \mathbb{P}\left(\mathcal{M}_{m \times(n+1)}(\mathbb{C})\right) .
$$

Then, $\mathcal{J}_{\mathbb{P}}(\Phi)$ equals the following quantity:

$$
2 \pi \nu\left[\mathbb{P}_{N-m-n m}(\mathbb{C})\right] \nu\left[\mathbb{P}_{n-m}(\mathbb{C})\right] \mathcal{D} \int_{0}^{1}\left(1-t^{2}\right)^{N-m-n m} t^{2 n m+2 m-1} \mathcal{I}_{\mathcal{M}}(\Phi, t) d t
$$

A first consequence is the following result. 
Corollary 4.2. For every polynomial system $h \in \mathbb{P}\left(\mathcal{H}_{(d)}^{m}\right)$, except for a measure zero set, the following equality holds:

$$
\nu\left[V_{\mathbb{P}}(h)\right]=\nu\left[\mathbb{P}_{n-m}(\mathbb{C})\right] \mathcal{D} .
$$

Proof. Apply Theorem 4.1 to the constant function $\Phi \equiv 1$. We obtain that

$$
\begin{gathered}
\int_{h \in \mathbb{P}\left(\mathcal{H}_{(d)}^{m}\right)} \nu\left[V_{\mathbb{P}}(h)\right] d \mathbb{P}\left(\mathcal{H}_{(d)}^{m}\right) \\
=2 \pi \nu\left[\mathbb{P}_{N-m-n m}(\mathbb{C})\right] \nu\left[\mathbb{P}_{n-m}(\mathbb{C})\right] \mathcal{D} \nu\left[\mathbb{P}_{n m+m-1}(\mathbb{C})\right] \\
\times \int_{0}^{1}\left(1-t^{2}\right)^{N-m-n m} t^{2 n m+2 m-1} d t .
\end{gathered}
$$

The value of this last integral is well known:

$$
\frac{1}{2} \frac{\Gamma(n m+m) \Gamma(N-m-n m+1)}{\Gamma(N+1)},
$$

where $\Gamma$ is the Gamma function. Now, using the fact that

$$
\nu\left[\mathbb{P}_{k}(\mathbb{C})\right]=\frac{\pi^{k}}{\Gamma(k+1)},
$$

for every nonnegative integer $k \in \mathbb{N}$, we obtain that

$$
\frac{1}{\nu\left[\mathbb{P}\left(\mathcal{H}_{(d)}^{m}\right)\right]} \int_{h \in \mathbb{P}\left(\mathcal{H}_{(d)}^{m}\right)} \nu\left[V_{\mathbb{P}}(h)\right] d \mathbb{P}\left(\mathcal{H}_{(d)}^{m}\right)=\nu\left[\mathbb{P}_{n-m}(\mathbb{C})\right] \mathcal{D}
$$

On the other hand, for almost all polynomial systems $h \in \mathbb{P}\left(\mathcal{H}_{(d)}^{m}\right)$, we have that $h$ is a regular value of the projection $p_{1}$ defined in Subsection 2.2 , Hence, $V_{\mathbb{P}}(h)$ is a smooth algebraic variety of complex dimension $n-m$, and from [Mum76, th. 5.22] (cf. also BP06a]) we conclude that

$$
\nu\left[V_{\mathbb{P}}(h)\right]=\nu\left[\mathbb{P}_{n-m}(\mathbb{C})\right] \operatorname{deg}\left(V_{\mathbb{P}}(h)\right),
$$

where $\operatorname{deg}(V)$ is the degree of $V$ in the sense of [Hei83. We conclude that

$$
\frac{1}{\nu\left[\mathbb{P}\left(\mathcal{H}_{(d)}^{m}\right)\right]} \int_{h \in \mathbb{P}\left(\mathcal{H}_{(d)}^{m}\right)} \operatorname{deg}\left(V_{\mathbb{P}}(h)\right) d \mathbb{P}\left(\mathcal{H}_{(d)}^{m}\right)=\mathcal{D}
$$

On the other hand, the Bézout inequality (cf. [Hei83) yields

$$
\operatorname{deg}\left(V_{\mathbb{P}}(h)\right) \leq \mathcal{D}, \quad \forall h \in \mathbb{P}\left(\mathcal{H}_{(d)}^{m}\right) .
$$

Thus, we conclude that $\operatorname{deg}\left(V_{\mathbb{P}}(h)\right)=\mathcal{D}$ for almost all $h \in \mathbb{P}\left(\mathcal{H}_{(d)}^{m}\right)$, and the corollary follows from (4.1).

The proof of Theorem 4.1 is divided into the following two subsections.

4.1. Some technical calculations. We recover the notation of Subsection 2.2. Thus, let $W$ be the incidence variety, and let $V_{e_{0}} \subseteq \mathbb{P}\left(\mathcal{H}_{(d)}^{m}\right)$ be the set of systems which have $e_{0}$ as a solution. We start with the following theorem, which uses the unitary invariance of the Riemannian structure of $\mathbb{P}\left(\mathcal{H}_{(d)}^{m}\right)$ defined in Subsection 2.1 . 
Theorem 4.3. Let $\phi: W \longrightarrow \mathbb{R}$ be an integrable mapping, such that for every $(h, \zeta) \in W$ and every unitary matrix $U \in \mathcal{U}_{n+1}$, the following equality holds:

$$
\phi(h, \zeta)=\phi\left(h \circ U, U^{-1} \zeta\right) .
$$

Let $\mathcal{J}$ be given by

$$
\mathcal{J}:=\int_{(h, \zeta) \in W} \phi(h, \zeta) N J_{(h, \zeta)} p_{1} d W
$$

Then, the following two equalities hold:

$$
\begin{gathered}
\mathcal{J}=\int_{h \in \mathbb{P}\left(\mathcal{H}_{(d)}^{m}\right)} \int_{\zeta \in V_{\mathbb{P}}(h)} \phi(h, \zeta) d V_{\mathbb{P}}(h) d \mathbb{P}\left(\mathcal{H}_{(d)}^{m}\right), \\
\mathcal{J}=\nu\left[\mathbb{P}_{n}(\mathbb{C})\right] \int_{h \in V_{e_{0}}} \phi\left(h, e_{0}\right) \frac{N J_{\left(h, e_{0}\right)} p_{1}}{N J_{\left(h, e_{0}\right)} p_{2}} d V_{e_{0}} .
\end{gathered}
$$

Proof. The first of the two equalities comes from Theorem 2.7 applied to $p_{1}$. As for the second one, also from Theorem 2.7] we have that

$$
\mathcal{J}=\int_{x \in \mathbb{P}_{n}(\mathbb{C})} \int_{h \in V_{x}} \phi(h, x) \frac{N J_{(h, x)} p_{1}}{N J_{(h, x)} p_{2}} d V_{x} d \mathbb{P}_{n}(\mathbb{C}) .
$$

Now, let $x \in \mathbb{P}_{n}(\mathbb{C})$ be any point and let $U \in \mathcal{U}_{n+1}$ be a unitary matrix such that $U e_{0}=x$. Then, the mapping sending $h$ to $h \circ U$ is an isometry from $V_{x}$ to $V_{e_{0}}$. Thus,

$$
\int_{h \in V_{x}} \phi(h, x) \frac{N J_{(h, x)} p_{1}}{N J_{(h, x)} p_{2}} d V_{x}=\int_{h \in V_{e_{0}}} \phi\left(h \circ U^{-1}, U e_{0}\right) \frac{N J_{\left(h \circ U^{-1}, U e_{0}\right)} p_{1}}{N J_{\left(h \circ U^{-1}, U e_{0}\right)} p_{2}} d V_{e_{0}} .
$$

Now, $\phi\left(h \circ U^{-1}, U e_{0}\right)=\phi\left(h, e_{0}\right)$. Also, observe that the mappings $\psi_{U}^{1}$ and $\psi_{U}^{2}$ defined as follows

$$
\begin{aligned}
& \psi_{U}^{1}: \quad W \quad \longrightarrow \quad W, \quad \psi_{U}^{2}: \mathbb{P}_{n}(\mathbb{C}) \longrightarrow \mathbb{P}_{n}(\mathbb{C}) \\
& (g, z) \mapsto\left(g \circ U^{-1}, U z\right) \quad \mapsto \quad \mapsto \quad U z
\end{aligned}
$$

are isometries. Moreover, they satisfy the conditions of Proposition 2.8. Thus,

$$
N J_{\left(h \circ U^{-1}, U e_{0}\right)} p_{2}=N J_{\left(h, e_{0}\right)} p_{2} .
$$

A similar argument with the mapping $\psi_{U}^{3}: \mathbb{P}\left(\mathcal{H}_{(d)}^{m}\right) \longrightarrow \mathbb{P}\left(\mathcal{H}_{(d)}^{m}\right)$ given as $\psi_{3}(h):=h \circ U^{-1}$ yields

$$
N J_{\left(h \circ U^{-1}, U e_{0}\right)} p_{1}=N J_{\left(h, e_{0}\right)} p_{1},
$$

and the theorem follows.

Lemma 4.4. Let $h \in V_{e_{0}}$ be such that $\operatorname{rank}\left(T_{e_{0}} h\right)=m$. Then, the following equalities hold:

$$
\begin{gathered}
N J_{\left(h, e_{0}\right)} p_{1}=\frac{1}{\operatorname{det}\left(I d_{m}+\left(\left(T_{e_{0}} h\right)^{\dagger}\right)^{*}\left(T_{e_{0}} h\right)^{\dagger}\right)}, \\
N J_{\left(h, e_{0}\right)} p_{2}=\frac{1}{\operatorname{det}\left(I d_{m}+\left(T_{e_{0}} h\right)\left(T_{e_{0}} h\right)^{*}\right)},
\end{gathered}
$$

where for any matrix $A, A^{\dagger}$ holds for the Moore-Penrose inverse of $A$, and $A^{*}$ holds for the Hermitian transpose of $A$. 
Proof. Recall that from Proposition 2.2

$$
T_{\left(h, e_{0}\right)} W \equiv\left\{\left(h^{\prime}, x\right) \in h^{\perp} \times e_{0}^{\perp}: h^{\prime}\left(e_{0}\right)+\left(T_{e_{0}} h\right) x=0\right\},
$$

where some representation of norm equal to 1 of $h$ has been chosen. Let $K_{1}:=$ $\operatorname{Ker}\left(d_{\left(h, e_{0}\right)} p_{1}\right)$ be the kernel of the tangent maaping at $\left(h, e_{0}\right)$. Then, $K_{1}=\{(0, x)$ : $\left.x \in \operatorname{Ker}\left(T_{e_{0}} h\right)\right\}$, and

$$
\begin{gathered}
N J_{\left(h, e_{0}\right)} p_{1}=N J_{(0,0)}\left(\left.\left(d_{\left(h, e_{0}\right)} p_{1}\right)\right|_{K_{1}^{\perp}}\right)=\frac{1}{N J_{(0,0)}\left(\left(\left.\left(d_{\left(h, e_{0}\right)} p_{1}\right)\right|_{K_{1}^{\perp}}\right)^{-1}\right)} \\
=\frac{1}{N J_{(0,0)}\left(\left(d_{\left(h, e_{0}\right)} p_{1}\right)^{\dagger}\right)} .
\end{gathered}
$$

Let $\beta$ be an orthonormal basis of $h^{\perp}$ such that the first $m$ elements of the basis are the systems

$$
\begin{gathered}
\beta_{1}:=\left(X_{0}^{d_{1}}, 0, \ldots, 0\right), \\
\vdots \\
\beta_{m}:=\left(0, \ldots, 0, X_{0}^{d_{m}}\right) .
\end{gathered}
$$

Observe that the first $m$ coordinates of any system $h^{\prime}:=\left[h_{1}^{\prime}, \ldots, h_{m}^{\prime}\right] \in \mathcal{H}_{(d)}^{m}$ in this basis are exactly $h^{\prime}\left(e_{0}\right)=\left(h_{1}^{\prime}\left(e_{0}\right), \ldots, h_{m}^{\prime}\left(e_{0}\right)\right)$. Moreover, the following properties hold:

- $\left(d_{\left(h, e_{0}\right)} p_{1}\right)^{\dagger}\left(\beta_{i}\right)=\left(\beta_{i}, x_{i}\right), x_{i}:=-\left(T_{e_{0}} h\right)^{\dagger}\left(e_{i}\right)$, for $1 \leq i \leq m$,

- $\left(d_{\left(h, e_{0}\right)} p_{1}\right)^{\dagger}(v)=(v, 0)$, for $v \in \beta, v \notin\left\{\beta_{1}, \ldots, \beta_{m}\right\}$.

Thus,

$$
N J_{(0,0)}\left(\left(d_{\left(h, e_{0}\right)} p_{1}\right)^{\dagger}\right)=\operatorname{det}\left(I d_{m}+\left(\left(T_{e_{0}} h\right)^{\dagger}\right)^{*}\left(T_{e_{0}} h\right)^{\dagger}\right) .
$$

As for $p_{2}$, observe that as above,

$$
N J_{\left(h, e_{0}\right)} p_{2}=\frac{1}{N J_{(0,0)}\left(\left(d_{\left(h, e_{0}\right)} p_{2}\right)^{\dagger}\right)} .
$$

Now, the following equality holds:

$$
\operatorname{Ker}\left(d_{\left(h, e_{0}\right)} p_{2}\right)^{\perp}=\left\{\left(h^{\prime}, 0\right): h^{\prime}\left(e_{0}\right)=0\right\}^{\perp}=\left\langle\beta_{1}, \ldots, \beta_{m}\right\rangle \times \mathbb{C}^{n},
$$

where $\left\langle\beta_{1}, \ldots, \beta_{m}\right\rangle$ stands for the linear subspace spanned by these vectors. Thus,

$$
\left(d_{\left(h, e_{0}\right)} p_{2}\right)^{\dagger}\left(e_{i}\right)=\left(h_{i}^{\prime}, e_{i}\right), 1 \leq i \leq n,
$$

where the first $m$ coordinates of $h_{i}^{\prime}$ in the basis $\beta$ are given by

$$
h_{i}^{\prime}:=-\left(T_{e_{0}} h\right) e_{i},
$$

and the rest of the coordinates equal 0 . Hence,

$$
N J_{(0,0)}\left(\left(d_{\left(h, e_{0}\right)} p_{2}\right)^{\dagger}\right)=\operatorname{det}\left(I d_{n}+\left(T_{e_{0}} h\right)^{*}\left(T_{e_{0}} h\right)\right)=\operatorname{det}\left(I d_{m}+\left(T_{e_{0}} h\right)\left(T_{e_{0}} h\right)^{*}\right),
$$

and the lemma follows.

Lemma 4.5. Let $h \in V_{e_{0}}$ be such that $\operatorname{rank}\left(T_{e_{0}} h\right)=m$. With the notation above, the following equality holds:

$$
\frac{N J_{\left(h, e_{0}\right)} p_{1}}{N J_{\left(h, e_{0}\right)} p_{2}}=\operatorname{det}\left(\left(T_{e_{0}} h\right)\left(T_{e_{0}} h\right)^{*}\right)
$$


Proof. From Lemma 4.4.

$$
\frac{N J_{\left(h, e_{0}\right)} p_{1}}{N J_{\left(h, e_{0}\right)} p_{2}}=\frac{\operatorname{det}\left(I d_{m}+B B^{*}\right)}{\operatorname{det}\left(I d_{m}+\left(B^{\dagger}\right)^{*} B^{\dagger}\right)},
$$

where $B:=T_{e_{0}} h \in \mathcal{M}_{m \times n}(\mathbb{C})$ is this matrix. Then,

$$
\frac{1}{\operatorname{det}\left(B B^{*}\right)} \frac{N J_{\left(h, e_{0}\right)} p_{1}}{N J_{\left(h, e_{0}\right)} p_{2}}=\frac{\operatorname{det}\left(I d_{m}+B B^{*}\right)}{\operatorname{det}\left(B B^{*}+B B^{*}\left(B^{\dagger}\right)^{*} B^{\dagger}\right)} .
$$

Now, $B B^{*}\left(B^{\dagger}\right)^{*} B^{\dagger}=B\left(B^{\dagger} B\right)^{*} B^{\dagger}$ and $B^{\dagger} B$ is selfadjoint. Moreover, $B B^{\dagger}=$ $I d_{m}$. Thus,

$$
\operatorname{det}\left(B B^{*}+B B^{*}\left(B^{\dagger}\right)^{*} B^{\dagger}\right)=\operatorname{det}\left(B B^{*}+B B^{\dagger} B B^{\dagger}\right)=\operatorname{det}\left(B B^{*}+I d_{m}\right),
$$

and the lemma follows.

Corollary 4.6. Let $\Phi:[0,+\infty] \longrightarrow[0,+\infty]$ be an integrable mapping. Then, the following equality holds:

$$
\begin{aligned}
& \int_{h \in \mathbb{P}\left(\mathcal{H}_{(d)}^{m}\right)} \int_{\zeta \in V_{\mathbb{P}}(h)} \Phi\left(\mu_{\text {norm }}^{m}(h, \zeta)\right) d V_{\mathbb{P}}(h) d \mathbb{P}\left(\mathcal{H}_{(d)}^{m}\right) \\
= & \nu\left[\mathbb{P}_{n}(\mathbb{C})\right] \int_{h \in V_{e_{0}}} \Phi\left(\mu_{\text {norm }}^{m}\left(h, e_{0}\right)\right) \operatorname{det}\left(\left(T_{e_{0}} h\right)\left(T_{e_{0}} h\right)^{*}\right) d V_{e_{0}} .
\end{aligned}
$$

Proof. Observe that for every element $(h, \zeta) \in W$ and for every unitary matrix $U \in \mathcal{U}_{n+1}(\mathbb{C})$, we have that

$$
\mu_{\text {norm }}^{m}(h, \zeta)=\mu_{\text {norm }}^{m}\left(h \circ U, U^{-1} \zeta\right) .
$$

Thus, the following equality also holds:

$$
\Phi\left(\mu_{\text {norm }}^{m}(h, \zeta)\right)=\Phi\left(\mu_{\text {norm }}^{m}\left(h \circ U, U^{-1} \zeta\right)\right) .
$$

The corollary follows from Theorem 4.3, applied to $\phi:=\Phi \circ \mu_{\text {norm }}^{m}$, and from Lemma 4.5

Corollary 4.7. Let $\Phi:[0,+\infty] \longrightarrow[0,+\infty]$ be an integrable mapping. Then, the following equality holds:

$$
\begin{aligned}
& \nu\left[\mathbb{P}_{n-m}(\mathbb{C})\right] \int_{M \in \mathbb{P}\left(\mathcal{M}_{m \times(n+1)}(\mathbb{C})\right)} \Phi\left(\kappa_{D}^{m}(M)\right) d \mathbb{P}\left(\mathcal{M}_{m \times(n+1)}(\mathbb{C})\right) \\
= & \nu\left[\mathbb{P}_{n}(\mathbb{C})\right] \int_{M \in \mathbb{P}\left(\mathcal{M}_{m \times n}(\mathbb{C})\right)} \Phi\left(\kappa_{D}^{m}(M)\right) \operatorname{det}\left(M M^{*}\right) \mathbb{P}\left(\mathcal{M}_{m \times n}(\mathbb{C})\right),
\end{aligned}
$$

where the representation of $M$ in the last integral is chosen such that $\|M\|_{F}=1$.

Proof. Apply Corollary 4.6 to the case that $(d):=(1, \ldots, 1) \in \mathbb{N}^{n}$. Then, the space $\mathbb{P}\left(\mathcal{H}_{(d)}^{m}\right)$ turns out to be $\mathbb{P}\left(\mathcal{M}_{m \times(n+1)}(\mathbb{C})\right)$, and the condition number $\mu_{\text {norm }}^{m}(M, \zeta)$, where $\zeta \neq 0$ is in the kernel of $M$, turns out to be exactly $\kappa_{D}^{m}(M)$. Hence, $\mu_{\text {norm }}^{m}(M, \zeta)$ does not depend on the solution $\zeta$, and Corollary 4.6 yields

$$
\begin{gathered}
\int_{M \in \mathbb{P}\left(\mathcal{M}_{m \times(n+1)}(\mathbb{C})\right)} \Phi\left(\kappa^{m}(M)\right) \nu[\operatorname{Ker}(M)] d \mathbb{P}\left(\mathcal{M}_{m \times(n+1)}(\mathbb{C})\right) \\
=\nu\left[\mathbb{P}_{n}(\mathbb{C})\right] \int_{M \in V_{e_{0}}} \Phi\left(\kappa_{D}^{m}(M) \operatorname{det}\left(\left(T_{e_{0}} M\right)\left(T_{e_{0}} M\right)^{*}\right)\right) d V_{e_{0}} .
\end{gathered}
$$


Now, in the linear case we have that

$$
V_{e_{0}}=\left\{M \in \mathbb{P}\left(\mathcal{M}_{m \times(n+1)}(\mathbb{C})\right): M e_{0}=0\right\}
$$

is a linear subspace of $\mathbb{P}\left(\mathcal{M}_{m \times(n+1)}(\mathbb{C})\right)$, which may obviously be identified with $\mathbb{P}\left(\mathcal{M}_{m \times n}(\mathbb{C})\right)$. In fact, a matrix belongs to $V_{e_{0}}$ if its first column is equal to zero. Moreover, under this identification, the value of $\kappa_{D}^{m}$, as defined in $\mathbb{P}\left(\mathcal{M}_{m \times(n+1)}(\mathbb{C})\right)$ and $\mathbb{P}\left(\mathcal{M}_{m \times n}(\mathbb{C})\right)$, does not vary. Finally, observe that for $M \in \mathbb{P}\left(\mathcal{M}_{m \times n}(\mathbb{C})\right)$, we have that $T_{e_{0}}(0 M)$ equals $M$ (for some fixed representation such that $\|M\|_{F}=1$ ). The corollary follows from the fact that $\nu[\operatorname{Ker}(M)]=\nu\left[\mathbb{P}_{n-m}(\mathbb{C})\right]$, for almost all $M \in \mathbb{P}\left(\mathcal{M}_{m \times(n+1)}(\mathbb{C})\right)$.

4.2. Proof of Theorem 4.1. We introduce some extra notation, which will only be used inside of this proof. Let $\hat{V}_{e_{0}}$ be the set defined as follows:

$$
\hat{V}_{e_{0}}:=\left\{h \in \mathcal{H}_{(d)}^{m}:\|h\|_{\Delta}=1, h\left(e_{0}\right)=0\right\},
$$

and let $\hat{L}_{e_{0}} \subseteq \mathcal{H}_{(d)}^{m}$ be the complex linear subspace of polynomial systems defined as follows:

$$
\hat{L}_{e_{0}}:=\left\{h \in \mathcal{H}_{(d)}^{m}: h_{i}=X_{0}^{d_{i}-1} \sum_{j=1}^{n} a_{i j} X_{j}, 1 \leq i \leq m\right\} .
$$

Let $\hat{V}_{e_{0}}, \hat{L}_{e_{0}}$ be endowed with the Riemannian structure inherited from that of $\mathcal{H}_{(d)}^{m}$. For any point $h \in \mathcal{H}_{(d)}^{m}, h\left(e_{0}\right)=0$, we denote by $\hat{T}_{e_{0}} h$ the restriction of the differential matrix to $e_{0}^{\perp}$. Namely,

$$
\hat{T}_{e_{0}} h:=\left.\left(d_{e_{0}} h\right)\right|_{e_{0}^{\perp}}, \quad \text { in the natural basis. }
$$

We consider the following mapping:

$$
\begin{aligned}
& \hat{\psi}_{e_{0}}: \hat{L}_{e_{0}} \longrightarrow \\
& h \mapsto \mathcal{M}_{m \times n}(\mathbb{C}) \\
& \operatorname{Diag}\left(d_{i}^{-1 / 2}\right) \hat{T}_{e_{0}} h,
\end{aligned}
$$

where $\operatorname{Diag}\left(d_{i}^{-1 / 2}\right):=\operatorname{Diag}\left(d_{1}^{-1 / 2}, \ldots, d_{m}^{-1 / 2}\right) \in \mathcal{M}_{m}(\mathbb{C})$ is this matrix. Some elementary calculations show that $\hat{\psi}_{e_{0}}$ is an isometry (cf. also BCSS98, Lemma 17, page 235]).

Let $\hat{\pi}: \hat{V}_{e_{0}} \longrightarrow \hat{L}_{e_{0}}$ be the orthogonal projection. Observe that $\hat{V}_{e_{0}}$ is a real Riemannian manifold of real dimension $2 N-2 m+1, \hat{L}_{e_{0}}$ is a complex subspace of $\mathcal{H}_{(d)}^{m}$ of complex dimension $n m$ and for every $h \in \hat{L}_{e_{0}},\|h\|_{\Delta}<1$, the set $\hat{\pi}^{-1}(h)$ is a sphere of real dimension $2 N-2 m+1-2 n m$ and radius $\left(1-\|h\|_{\Delta}^{2}\right)^{1 / 2}$. Thus, the $(2 N-2 m+1-2 n m)$-dimensional volume of $\hat{\pi}^{-1}(h)$ is

$$
\nu\left[\hat{\pi}^{-1}(h)\right]=\left(1-\|h\|_{\Delta}^{2}\right)^{N-m-n m+1 / 2} 2 \pi \nu\left[\mathbb{P}_{N-m-n m}(\mathbb{C})\right] .
$$

Moreover, some elementary calculations lead to the following expression:

$$
N J_{h} \hat{\pi}=\left(1-\|\hat{\pi}(h)\|_{\Delta}^{2}\right)^{1 / 2} .
$$

We have denoted by $\mathcal{J}_{\mathbb{P}}(\Phi)$ the integral in the space of polynomial systems. Namely,

$$
\mathcal{J}_{\mathbb{P}}(\Phi):=\int_{h \in \mathbb{P}\left(\mathcal{H}_{(d)}^{m}\right)} \int_{\zeta \in V_{\mathbb{P}}(h)} \Phi\left(\mu_{\text {norm }}^{m}(h, \zeta)\right) d V_{\mathbb{P}}(h) d \mathbb{P}\left(\mathcal{H}_{(d)}^{m}\right)
$$


From Corollary 4.6 we have that

$$
\mathcal{J}_{\mathbb{P}}(\Phi)=\nu\left[\mathbb{P}_{n}(\mathbb{C})\right] \int_{h \in V_{e_{0}}} \Phi\left(\mu_{\text {norm }}^{m}\left(h, e_{0}\right)\right) \operatorname{det}\left(\left(T_{e_{0}} h\right)\left(T_{e_{0}} h\right)^{*}\right) d V_{e_{0}} .
$$

Now, as observed in [BCSS98, th.1, page 256],

$$
\begin{aligned}
& \nu\left[\mathbb{P}_{n}(\mathbb{C})\right] \int_{h \in V_{e_{0}}} \Phi\left(\mu_{\text {norm }}^{m}\left(h, e_{0}\right)\right) \operatorname{det}\left(\left(T_{e_{0}} h\right)\left(T_{e_{0}} h\right)^{*}\right) d V_{e_{0}} \\
= & \frac{\nu\left[\mathbb{P}_{n}(\mathbb{C})\right]}{2 \pi} \int_{h \in \hat{V}_{e_{0}}} \Phi\left(\mu_{\text {norm }}^{m}\left(h, e_{0}\right)\right) \operatorname{det}\left(\left(\hat{T}_{e_{0}} h\right)\left(\hat{T}_{e_{0}} h\right)^{*}\right) d \hat{V}_{e_{0}} .
\end{aligned}
$$

From Theorem 2.7, this last equals

$$
\frac{\nu\left[\mathbb{P}_{n}(\mathbb{C})\right]}{2 \pi} \int_{h \in \hat{L}_{e_{0}}} \int_{h^{\prime} \in \hat{\pi}^{-1}(h)} \Phi\left(\mu_{\text {norm }}^{m}\left(g, e_{0}\right)\right) \frac{\operatorname{det}\left(\left(\hat{T}_{e_{0}} h^{\prime}\right)\left(\hat{T}_{e_{0}} h^{\prime}\right)^{*}\right)}{\left(1-\|h\|_{\Delta}^{2}\right)^{1 / 2}} d \hat{\pi}^{-1}(h) d \hat{L}_{e_{0}} .
$$

Now, observe that if $h^{\prime} \in \hat{\pi}^{-1}(h)$, then

$$
\mu_{\text {norm }}^{m}\left(h^{\prime}, e_{0}\right)=\frac{\kappa_{D}^{m}\left(\hat{\psi}_{e_{0}}(h)\right)}{\left\|\hat{\psi}_{e_{0}}(h)\right\|_{F}}, \hat{T}_{e_{0}} h^{\prime}=\hat{T}_{e_{0}} h .
$$

We conclude that

$$
\mathcal{J}_{\mathbb{P}}(\Phi)=\frac{\nu\left[\mathbb{P}_{n}(\mathbb{C})\right]}{2 \pi} \int_{\substack{h \in \hat{L}_{e_{0}} \\\|h\|_{\Delta} \leq 1}} \nu\left[\hat{\pi}^{-1}(h)\right] \Phi\left(\frac{\kappa_{D}^{m}\left(\hat{\psi}_{e_{0}}(h)\right)}{\left\|\hat{\psi}_{e_{0}}(h)\right\|_{F}}\right) \frac{\operatorname{det}\left(\left(\hat{T}_{e_{0}} h\right)\left(\hat{T}_{e_{0}} h\right)^{*}\right)}{\left(1-\|h\|_{\Delta}^{2}\right)^{1 / 2}} d \hat{L}_{e_{0}} .
$$

From identity (4.2),

$$
\begin{gathered}
\mathcal{J}_{\mathbb{P}}(\Phi)=\nu\left[\mathbb{P}_{n}(\mathbb{C})\right] \nu\left[\mathbb{P}_{N-m-n m}(\mathbb{C})\right] \\
\times \int_{\substack{h \in \hat{L}_{e_{0}} \\
\|h\|_{\Delta \leq 1}}}\left(1-\|h\|_{\Delta}^{2}\right)^{N-m-n m} \Phi\left(\frac{\kappa_{D}^{m}\left(\hat{\psi}_{e_{0}}(h)\right)}{\left\|\hat{\psi}_{e_{0}}(h)\right\|_{F}}\right) \operatorname{det}\left(\left(\hat{T}_{e_{0}} h\right)\left(\hat{T}_{e_{0}} h\right)^{*}\right) d \hat{L}_{e_{0}} .
\end{gathered}
$$

Then, Theorem 2.7 applied to $\hat{\psi}_{e_{0}}$ yields

$$
\begin{aligned}
& \int_{\substack{h \in \hat{L}_{e_{0}} \\
\|h\|_{\Delta \leq 1}}}\left(1-\|h\|_{\Delta}^{2}\right)^{N-m-n m} \Phi\left(\frac{\kappa_{D}^{m}\left(\hat{\psi}_{e_{0}}(h)\right)}{\left\|\hat{\psi}_{e_{0}}(h)\right\|_{F}}\right) \operatorname{det}\left(\left(\hat{T}_{e_{0}} h\right)\left(\hat{T}_{e_{0}} h\right)^{*}\right) d \hat{L}_{e_{0}} \\
= & \operatorname{det}\left(\operatorname{Diag}\left(d_{i}\right)\right) \int_{\substack{M \in \mathcal{M}_{m \times n}(\mathbb{C}) \\
\|M\|_{F} \leq 1}}\left(1-\|M\|_{F}^{2}\right)^{N-m-n m} \Phi\left(\frac{\kappa_{D}^{m}(M)}{\|M\|_{F}}\right) \operatorname{det}\left(M M^{*}\right) d \mathcal{M}_{m \times n}(\mathbb{C}) \\
= & \mathcal{D} \int_{\substack{M \in \mathcal{M}_{m \times n}(\mathbb{C}) \\
\|M\|_{F} \leq 1}}\left(1-\|M\|_{F}^{2}\right)^{N-m-n m} \Phi\left(\frac{\kappa_{D}^{m}(M)}{\|M\|_{F}}\right) \operatorname{det}\left(M M^{*}\right) d \mathcal{M}_{m \times n}(\mathbb{C}) .
\end{aligned}
$$

In polar coordinates, this last equals

$$
\begin{gathered}
\mathcal{D} \int_{0}^{1}\left(1-t^{2}\right)^{N-m-n m} \int_{\|M\|_{F}=t} \Phi\left(\frac{\kappa_{D}^{m}(M)}{t}\right) \operatorname{det}\left(M M^{*}\right) d S^{t}\left(\mathcal{M}_{m \times n}(\mathbb{C})\right) d t \\
=\mathcal{D} \int_{0}^{1}\left(1-t^{2}\right)^{N-m-n m} t^{2 m n+2 m-1} \\
\times \int_{\|M\|_{F}=1} \Phi\left(\frac{\kappa_{D}^{m}(M)}{t}\right) \operatorname{det}\left(M M^{*}\right) d S^{1}\left(\mathcal{M}_{m \times n}(\mathbb{C})\right) d t
\end{gathered}
$$


Now, observe that for every choice of $t \in[0,1]$,

$$
\begin{gathered}
\int_{\|M\|_{F}=1} \Phi\left(\frac{\kappa_{D}^{m}(M)}{t}\right) \operatorname{det}\left(M M^{*}\right) d S^{1}\left(\mathcal{M}_{m \times n}(\mathbb{C})\right) \\
=2 \pi \int_{M \in \mathbb{P}\left(\mathcal{M}_{m \times n}(\mathbb{C})\right)} \Phi\left(\frac{\kappa_{D}^{m}(M)}{t}\right) \operatorname{det}\left(M M^{*}\right) d \mathbb{P}\left(\mathcal{M}_{m \times n}(\mathbb{C})\right),
\end{gathered}
$$

where the representation $M$ in the last formula is chosen such that $\|M\|_{F}=1$. Let

$$
\begin{array}{ccc}
\Phi_{t}:[0,+\infty] & \longrightarrow & {[0,+\infty]} \\
s & \mapsto & \Phi\left(\frac{s}{t}\right)
\end{array}
$$

be this positive mapping. Then,

$$
\begin{aligned}
& 2 \pi \int_{M \in \mathbb{P}\left(\mathcal{M}_{m \times n}(\mathbb{C})\right)} \Phi\left(\frac{\kappa_{D}^{m}(M)}{t}\right) \operatorname{det}\left(M M^{*}\right) d \mathbb{P}\left(\mathcal{M}_{m \times n}(\mathbb{C})\right) \\
= & 2 \pi \int_{M \in \mathbb{P}\left(\mathcal{M}_{m \times n}(\mathbb{C})\right)} \Phi_{t}\left(\kappa_{D}^{m}(M)\right) \operatorname{det}\left(M M^{*}\right) d \mathbb{P}\left(\mathcal{M}_{m \times n}(\mathbb{C})\right),
\end{aligned}
$$

and from Corollary 4.7, this last equals

$$
2 \pi \frac{\nu\left[\mathbb{P}_{n-m}(\mathbb{C})\right]}{\nu\left[\mathbb{P}_{n}(\mathbb{C})\right]} \int_{M \in \mathbb{P}\left(\mathcal{M}_{m \times(n+1)}(\mathbb{C})\right)} \Phi_{t}\left(\kappa_{D}^{m}(M)\right) d \mathbb{P}\left(\mathcal{M}_{m \times(n+1)}(\mathbb{C})\right) .
$$

We have thus proved that $\mathcal{J}_{\mathbb{P}}(\Phi)$ equals

$$
\begin{gathered}
2 \pi \nu\left[\mathbb{P}_{n-m}(\mathbb{C})\right] \nu\left[\mathbb{P}_{N-m-n m}(\mathbb{C})\right] \mathcal{D} \int_{0}^{1}\left(1-t^{2}\right)^{N-m-n m} t^{2 m n+2 m-1} \\
\times \int_{M \in \mathbb{P}\left(\mathcal{M}_{m \times(n+1)}(\mathbb{C})\right)} \Phi_{t}\left(\kappa_{D}^{m}(M)\right) d \mathbb{P}\left(\mathcal{M}_{m \times(n+1)}(\mathbb{C})\right),
\end{gathered}
$$

and the theorem follows.

\section{The average value of $\mu_{\mathrm{av}}^{m}$}

The aim of this section is to prove Theorem 1.4. We reproduce the technical version of this statement here.

Theorem 5.1. Let $m \geq 2$, and assume there exists some $i, 1 \leq i \leq m$, such that $d_{i}>1$. Then, the expected value of the condition number $\mu_{\mathrm{av}}^{m}$ satisfies

$$
\mathrm{E}_{\mathcal{P}(d)}^{m}\left[\mu_{\mathrm{av}}^{m}\right] \leq 3 m \sqrt{n N} .
$$

Moreover, if $m=1$, we have that

$$
\mathrm{E}_{\mathcal{P}_{(d)}^{1}}\left[\mu_{\mathrm{av}}^{1}\right]=\frac{\Gamma(N+1) \Gamma(n+1 / 2)}{\Gamma(N+1 / 2) \Gamma(n+1)} .
$$

Proof. From identity (2.4), the expected value $\mathrm{E}_{\mathcal{P}_{(d)}^{m}}\left[\mu_{\mathrm{av}}^{m}\right]$ (for the Gaussian distribution) satisfies

$$
\mathrm{E}_{\mathcal{P}_{(d)}^{m}}\left[\mu_{\mathrm{av}}^{m}\right]=\mathrm{E}_{\mathbb{P}\left(\mathcal{P}_{(d)}^{m}\right)}\left[\mu_{\mathrm{av}}^{m}\right]=\mathrm{E}_{\mathbb{P}\left(\mathcal{H}_{(d)}^{m}\right)}\left[\mu_{\mathrm{av}}^{m}\right]
$$

Now, this last quantity equals

$$
\frac{1}{\nu\left[\mathbb{P}\left(\mathcal{H}_{(d)}^{m}\right)\right]} \int_{h \in \mathbb{P}\left(\mathcal{H}_{(d)}^{m}\right)} \frac{1}{\nu\left[V_{\mathbb{P}}(h)\right]} \int_{\zeta \in V_{\mathbb{P}}(h)} \mu_{\text {norm }}^{m}(h, \zeta) d V_{\mathbb{P}}(h) d \mathbb{P}\left(\mathcal{H}_{(d)}^{m}\right) .
$$


Hence, we define the following quantity:

$$
\mathcal{K}_{(d)}:=\int_{h \in \mathbb{P}\left(\mathcal{H}_{(d)}^{m}\right)} \int_{\zeta \in V_{\mathbb{P}}(h)} \mu_{\text {norm }}^{m}(h, \zeta) d V_{\mathbb{P}}(h) d \mathbb{P}\left(\mathcal{H}_{(d)}^{m}\right) .
$$

From Corollary 4.2

$$
\mathrm{E}_{\mathbb{P}\left(\mathcal{H}_{(d)}^{m}\right)}\left[\mu_{\mathrm{av}}^{m}\right]=\frac{\mathcal{K}_{(d)}}{\nu\left[\mathbb{P}\left(\mathcal{H}_{(d)}^{m}\right)\right] \nu\left[\mathbb{P}_{n-m}(\mathbb{C})\right] \mathcal{D}} .
$$

Let us calculate a bound for $\mathcal{K}_{(d)}$. From Theorem 4.1.

$$
\begin{aligned}
\mathcal{K}_{(d)}=2 \pi \nu\left[\mathbb{P}_{N-m-n m}(\mathbb{C})\right] \nu\left[\mathbb{P}_{n-m}(\mathbb{C})\right] \mathcal{D} \int_{0}^{1}\left(1-t^{2}\right)^{N-m-n m} t^{2 n m+2 m-2} d t \\
\times \int_{M \in \mathbb{P}\left(\mathcal{M}_{m \times(n+1)}(\mathbb{C})\right)} \kappa_{D}^{m}(M) d \mathbb{P}\left(\mathcal{M}_{m \times(n+1)}(\mathbb{C})\right) .
\end{aligned}
$$

Now, observe that

$$
\int_{0}^{1}\left(1-t^{2}\right)^{N-m-n m} t^{2 n m+2 m-2} d t=\frac{1}{2} \frac{\Gamma(N-m-n m+1) \Gamma(n m+m-1 / 2)}{\Gamma(N+1 / 2)} .
$$

Hence, we have that

$$
\mathcal{K}_{(d)}=\nu\left[\mathbb{P}_{n-m}(\mathbb{C})\right] \mathcal{D} \pi^{N} \frac{\Gamma(n m+m-1 / 2)}{\Gamma(N+1 / 2) \Gamma(n m+m)} \mathrm{E}_{\mathbb{P}\left(\mathcal{M}_{m \times(n+1)}(\mathbb{C})\right)}\left[\kappa_{D}^{m}\right],
$$

where E stands for expectation. Thus,

$$
\mathrm{E}_{\mathbb{P}\left(\mathcal{H}_{(d)}^{m}\right)}\left[\mu_{\mathrm{av}}^{m}\right]=\frac{\Gamma(N+1) \Gamma(n m+m-1 / 2)}{\Gamma(N+1 / 2) \Gamma(n m+m)} \mathrm{E}_{\mathbb{P}\left(\mathcal{M}_{m \times(n+1)}(\mathbb{C})\right)}\left[\kappa_{D}^{m}\right] .
$$

The case $m=1$ of the theorem follows from Corollary 2.5. As for the case that $m \geq 2$, also from Corollary 2.5 we have that

$$
\mathrm{E}_{\mathbb{P}\left(\mathcal{H}_{(d)}^{m}\right)}\left[\mu_{\mathrm{av}}^{m}\right] \leq \frac{\Gamma(N+1) \Gamma(n m+m-1 / 2)}{\Gamma(N+1 / 2) \Gamma(n m+m)} \frac{2^{1 / 4} \mathrm{em}^{3 / 2}(n+1)}{n-m+3 / 2} .
$$

From Gautschi's inequalities (see [EGP00, Th. 3] for very sharp bounds), we know that for $x>0$,

$$
\sqrt{x+1 / 4} \leq \frac{\Gamma(x+1)}{\Gamma(x+1 / 2)} \leq \sqrt{x+1 / \pi} .
$$

Thus,

$$
\mathrm{E}_{\mathbb{P}\left(\mathcal{H}_{(d)}^{m}\right)}\left[\mu_{\mathrm{av}}^{m}\right] \leq 2^{1 / 4} e \sqrt{N+1 / \pi} \frac{m^{3 / 2}(n+1)}{(n-m+3 / 2) \sqrt{n m+m-3 / 4}} .
$$

Now, some elementary calculations show that this last quantity is smaller than

$$
3 m \sqrt{n N},
$$

for every choice of $n \geq m \geq 2$. In fact, observe that, as $d_{i}>1$ for some $i, \leq i \leq m$, we have that

$$
N>n m .
$$


Then, we have that

$$
\begin{gathered}
\frac{1}{3 m \sqrt{n N}} 2^{1 / 4} e \sqrt{N+1 / \pi} \frac{m^{3 / 2}(n+1)}{(n-m+3 / 2) \sqrt{n m+m-3 / 4}} \\
\leq \frac{2^{1+1 / 4} e}{9} \sqrt{1+\frac{1}{\pi N}} \sqrt{1+\frac{1}{n}} \sqrt{\frac{m n+m}{n m+m-3 / 4}} \\
\quad \leq \frac{2^{1+1 / 4} e}{9} \sqrt{1+\frac{1}{4 \pi}} \sqrt{1+\frac{1}{2}} \sqrt{\frac{6}{6-3 / 4}}<1 .
\end{gathered}
$$

Thus, we obtain that

$$
\mathrm{E}_{\mathbb{P}\left(\mathcal{H}_{(d)}^{m}\right)}\left[\mu_{\mathrm{av}}^{m}\right] \leq 3 m \sqrt{n N}
$$

as wanted.

\section{The average Value of $\mu_{\mathrm{worst}}^{m}$}

In this section we prove Theorem 1.3 , We start with the following estimation.

Corollary 6.1. Let $(d)=\left(d_{1}, \ldots, d_{m}\right)$ be such that $d_{i}>1$ for some $i, 1 \leq i \leq m$. Let $\varepsilon>0$ be a positive real number. Then, the following inequality holds:

$$
\begin{aligned}
\frac{1}{\nu\left[\mathbb{P}\left(\mathcal{H}_{(d)}^{m}\right)\right]} & \int_{h \in \mathbb{P}\left(\mathcal{H}_{(d)}^{m}\right)} \nu\left[\zeta \in V_{\mathbb{P}}(h): \mu_{\text {norm }}^{m}(h, \zeta)>\varepsilon^{-1}\right] d \mathbb{P}\left(\mathcal{H}_{(d)}^{m}\right) \\
& \leq \nu\left[\mathbb{P}_{n-m}(\mathbb{C})\right] \mathcal{D}(e m \sqrt{n N} \varepsilon)^{2(n-m+2)} .
\end{aligned}
$$

Proof. We apply Theorem 4.1 to the function $\Phi_{\varepsilon}:[0,+\infty] \longrightarrow[0,+\infty]$ defined as

$$
\Phi_{\varepsilon}(s):= \begin{cases}1 & \text { if } s>\varepsilon^{-1} \\ 0 & \text { otherwise }\end{cases}
$$

We conclude that

$$
\begin{gathered}
I_{\varepsilon}^{m}:=\int_{h \in \mathbb{P}\left(\mathcal{H}_{(d)}^{m}\right)} \nu\left[\zeta \in V_{\mathbb{P}}(h): \mu_{\text {norm }}^{m}(h, \zeta)>\varepsilon^{-1}\right] d \mathbb{P}\left(\mathcal{H}_{(d)}^{m}\right) \\
=2 \pi \nu\left[\mathbb{P}_{N-m-n m}(\mathbb{C})\right] \nu\left[\mathbb{P}_{n-m}(\mathbb{C})\right] \mathcal{D} \\
\times \int_{0}^{1}\left(1-t^{2}\right)^{N-m-n m} t^{2 n m+2 m-1} \nu\left[M \in \mathbb{P}\left(\mathcal{M}_{m \times(n+1)}(\mathbb{C})\right): \kappa_{D}^{m}(M)>\varepsilon^{-1} t\right] d t .
\end{gathered}
$$

Let $m=1$. Then, from Lemma 2.3 .

$$
\frac{1}{\nu\left[\mathbb{P}_{n}(\mathbb{C})\right]} \nu\left[M \in \mathbb{P}\left(\mathcal{M}_{1 \times(n+1)}(\mathbb{C})\right): \kappa_{D}^{1}(M)>\varepsilon^{-1} t\right]= \begin{cases}1 & \text { if } t<\varepsilon \\ 0 & \text { otherwise. }\end{cases}
$$

Thus,

$$
\begin{gathered}
I_{\varepsilon}^{1}=2 \pi \nu\left[\mathbb{P}_{N-1-n}(\mathbb{C})\right] \nu\left[\mathbb{P}_{n-1}(\mathbb{C})\right] \nu\left[\mathbb{P}_{n}(\mathbb{C})\right] \mathcal{D} \int_{0}^{\varepsilon}\left(1-t^{2}\right)^{N-1-n} t^{2 n+1} d t \\
\leq 2 \pi \nu\left[\mathbb{P}_{N-1-n}(\mathbb{C})\right] \nu\left[\mathbb{P}_{n-1}(\mathbb{C})\right] \nu\left[\mathbb{P}_{n}(\mathbb{C})\right] \mathcal{D} \int_{0}^{\varepsilon} t^{2 n+1} d t \\
=2 \pi \nu\left[\mathbb{P}_{N-1-n}(\mathbb{C})\right] \nu\left[\mathbb{P}_{n-1}(\mathbb{C})\right] \nu\left[\mathbb{P}_{n}(\mathbb{C})\right] \mathcal{D} \frac{\varepsilon^{2 n+2}}{2 n+2}
\end{gathered}
$$


Hence,

$$
\frac{1}{\nu\left[\mathbb{P}\left(\mathcal{H}_{(d)}^{1}\right)\right]} I_{\varepsilon}^{1} \leq \nu\left[\mathbb{P}_{n-1}(\mathbb{C})\right] \mathcal{D}\left(\begin{array}{c}
N \\
n+1
\end{array}\right) \varepsilon^{2 n+2} .
$$

In particular, the bound of the corollary follows for $m=1$. Now, let $m \geq 2$. Also from Lemma 2.3, we know that

$$
\begin{gathered}
\frac{1}{\nu\left[\mathbb{P}_{n m+m-1}(\mathbb{C})\right]} \nu\left[M \in \mathbb{P}\left(\mathcal{M}_{m \times(n+1)}(\mathbb{C})\right): \kappa_{D}^{m}(M)>\varepsilon^{-1} t\right] \\
\leq 2\left(\frac{e m^{3 / 2}(n+1)}{n-m+2} \frac{\varepsilon}{t}\right)^{2(n-m+2)}
\end{gathered}
$$

Hence, $I_{\varepsilon}^{m}$ is at most

$$
\begin{aligned}
4 \pi \nu\left[\mathbb{P}_{N-m-n m}(\mathbb{C})\right] \nu\left[\mathbb{P}_{n-m}(\mathbb{C})\right] \mathcal{D} \nu\left[\mathbb{P}_{n m+m-1}(\mathbb{C})\right]\left(\frac{e m^{3 / 2}(n+1)}{n-m+2} \varepsilon\right)^{2(n-m+2)} \\
\times \int_{0}^{1}\left(1-t^{2}\right)^{N-m-n m} t^{2 n m+4 m-2 n-5} d t .
\end{aligned}
$$

This last integral equals

$$
\frac{1}{2} \frac{\Gamma(N-m-n m+1) \Gamma(n m+2 m-n-2)}{\Gamma(N+m-n-1)} .
$$

We conclude that

$$
\frac{1}{\nu\left[\mathbb{P}\left(\mathcal{H}_{(d)}^{m}\right)\right]} I_{\varepsilon}^{m} \leq 2 \nu\left[\mathbb{P}_{n-m}(\mathbb{C})\right] \mathcal{D}\left(\frac{e m^{3 / 2}(n+1)}{n-m+2} \varepsilon\right)^{2(n-m+2)} \vartheta(N, n, m),
$$

where

$$
\vartheta(N, n, m):=\frac{\Gamma(N+1) \Gamma(n m+2 m-n-2)}{\Gamma(N+m-n-1) \Gamma(n m+m)} .
$$

Finally, observe that

$$
\vartheta(N, n, m) \leq\left(\frac{N}{(n+2)(m-1)}\right)^{n-m+2} .
$$

The estimation of the corollary follows from the fact that

$$
2\left(\frac{e m^{3 / 2}(n+1)}{(n-m+2) \sqrt{(n+2)(m-1)}}\right)^{2(n-m+2)} \leq(e m \sqrt{n})^{2(n-m+2)},
$$

for every choice of $n \geq m \geq 2$. This last assertion can be verified by some elementary calculations.

Proposition 6.2. Let $h \in \mathbb{P}\left(\mathcal{H}_{(d)}^{m}\right), \zeta \in V_{\mathbb{P}}(h)$ be such that $\mu_{\mathrm{norm}}^{m}(h, \zeta)<\infty$. Let $\zeta^{\prime} \in V_{\mathbb{P}}(h)$ be another solution of $h$ such that

$$
u:=d_{\mathbb{P}}\left(\zeta^{\prime}, \zeta\right) \mu_{\text {norm }}^{m}(h, \zeta) \frac{\sqrt{2} d^{3 / 2}}{2}<1-\sqrt{2} / 2 .
$$

Then, the following inequality holds:

$$
\mu_{\text {norm }}^{m}\left(h, \zeta^{\prime}\right) \leq \frac{(1-u)^{2}}{2 u^{2}-4 u+1} \mu_{\text {norm }}^{m}(h, \zeta) .
$$


Proof. We denote by $\bar{h}, \bar{\zeta}, \bar{\zeta}^{\prime}$ some fixed representations of $h, \zeta, \zeta^{\prime}$ such that $\|\bar{h}\|_{\Delta}=$ $\|\bar{\zeta}\|_{2}=\left\|\bar{\zeta}^{\prime}\right\|_{2}=1$. Moreover, we can choose representatives such that

$$
\left\langle\bar{\zeta}, \bar{\zeta}^{\prime}\right\rangle_{2} \in \mathbb{R}^{0,+} .
$$

Then, observe that $T_{\bar{\zeta}} \bar{h}\left(T_{\bar{\zeta}} \bar{h}\right)^{\dagger}$ is the identity map. Hence,

$$
\begin{gathered}
\mu_{\text {norm }}^{m}\left(h, \zeta^{\prime}\right)=\left\|\left(T_{\bar{\zeta}^{\prime}} \bar{h}\right)^{\dagger} \operatorname{Diag}\left(d_{i}^{1 / 2}\right)\right\|_{2} \\
\leq\left\|\left(T_{\bar{\zeta}^{\prime}} \bar{h}\right)^{\dagger} T \bar{\zeta} \bar{h}\right\|_{2}\left\|\left(T_{\bar{\zeta}} \bar{h}\right)^{\dagger} \operatorname{Diag}\left(d_{i}^{1 / 2}\right)\right\|_{2}=\left\|\left(T_{\bar{\zeta}^{\prime}} \bar{h}\right)^{\dagger} T_{\bar{\zeta}} \bar{h}\right\|_{2} \mu_{\text {norm }}^{m}(h, \zeta) .
\end{gathered}
$$

Hence, it suffices to prove that in the conditions of the lemma, the following inequality holds:

Now, from Lemma 3.1

$$
\left\|\left(T_{\bar{\zeta}^{\prime}} \bar{h}\right)^{\dagger} T_{\bar{\zeta}} \bar{h}\right\|_{2} \leq \frac{(1-u)^{2}}{2 u^{2}-4 u+1} .
$$

$$
\left\|\left(T_{\bar{\zeta}^{\prime}} \bar{h}\right)^{\dagger} T_{\bar{\zeta}} \bar{h}\right\|_{2}=\left\|\left.\left(\left.\left(d_{\bar{\zeta}^{\prime}} \bar{h}\right)\right|_{\left(\bar{\zeta}^{\prime}\right)^{\perp}}\right)^{\dagger} d_{\bar{\zeta}} \bar{h}\right|_{(\bar{\zeta})^{\perp}}\right\|_{2}=\left\|\left(d_{\bar{\zeta}^{\prime}} \bar{h}\right)^{\dagger} d_{\bar{\zeta}} \bar{h}\right\|_{2}
$$

Let $\gamma(\bar{h}, \bar{\zeta})$ be the affine invariant defined in Section 2.1. considering $\bar{h}$ as a polynomial in $X_{0}, \ldots, X_{n}$. Namely,

$$
\gamma(\bar{h}, \bar{\zeta}):=\sup _{k \geq 2}\left\|\left(d_{\bar{\zeta}} \bar{h}\right)^{\dagger} \frac{d_{\bar{\zeta}}^{(k)} \bar{h}}{k !}\right\|_{2}^{1 /(k-1)}
$$

if $d_{\bar{\zeta}} \bar{h}$ is surjective. From Lemma 3.1. we have that

$$
\gamma(\bar{h}, \bar{\zeta})=\gamma_{0}(h, \zeta)
$$

where $\gamma_{0}$ is as defined in Section 3 . Hence, from Lemma 3.2 we have

$$
\gamma(\bar{h}, \bar{\zeta}) \leq \mu_{\text {norm }}^{m}(h, \zeta) \frac{d^{3 / 2}}{2} .
$$

On the other hand, the following inequality holds:

$$
\left\|\bar{\zeta}-\bar{\zeta}^{\prime}\right\|_{2}=\sqrt{2}\left(1-\left\langle\bar{\zeta}, \bar{\zeta}^{\prime}\right\rangle_{2}\right)^{1 / 2}=\sqrt{2}\left(1-\sqrt{1-d_{\mathbb{P}}\left(\zeta, \zeta^{\prime}\right)^{2}}\right)^{1 / 2} \leq \sqrt{2} d_{\mathbb{P}}\left(\zeta, \zeta^{\prime}\right) .
$$

Hence, we conclude that

$$
\left\|\bar{\zeta}-\bar{\zeta}^{\prime}\right\|_{2} \gamma(\bar{h}, \bar{\zeta}) \leq \sqrt{2} d_{\mathbb{P}}\left(\zeta, \zeta^{\prime}\right) \mu_{\text {norm }}^{m}(h, \zeta) \frac{d^{3 / 2}}{2}=u .
$$

Finally, from [SS96, pg. 20] or [Ded06, Chap. 5] we know that this implies

$$
\left\|\left(d_{\bar{\zeta}^{\prime}} \bar{h}\right)^{\dagger} d \bar{\zeta} \bar{h}\right\| \leq \frac{(1-u)^{2}}{2 u^{2}-4 u+1}
$$

and the lemma follows.

Corollary 6.3. Let $\varepsilon>0, s>1$ be two positive real numbers. Let $h \in \mathbb{P}\left(\mathcal{H}_{(d)}^{m}\right)$, $\zeta^{\prime} \in V_{\mathbb{P}}(h)$ be such that $1 / \varepsilon<\mu_{\text {norm }}^{m}\left(h, \zeta^{\prime}\right)<+\infty$. Let $\zeta \in V_{\mathbb{P}}(h)$ be another solution of $h$ such that

$$
d_{\mathbb{P}}\left(\zeta^{\prime}, \zeta\right) \leq \frac{\sqrt{2} \varepsilon}{d^{3 / 2}} s\left(1-\sqrt{\frac{s}{2 s-1}}\right) .
$$

Then, the following inequality holds:

$$
\mu_{\text {norm }}^{m}(h, \zeta)>\frac{1}{s \varepsilon} .
$$


Proof. Assume that

Then, we have that

$$
\mu_{\text {norm }}^{m}(h, \zeta) \leq \frac{1}{s \varepsilon}
$$

$$
\begin{aligned}
u:=d_{\mathbb{P}}\left(\zeta^{\prime}, \zeta\right) \mu_{\text {norm }}^{m} & (h, \zeta) \frac{\sqrt{2} d^{3 / 2}}{2} \leq \frac{\sqrt{2} \varepsilon}{d^{3 / 2}} s\left(1-\sqrt{\frac{s}{2 s-1}}\right) \frac{1}{s \varepsilon} \frac{\sqrt{2} d^{3 / 2}}{2} \\
= & \left(1-\sqrt{\frac{s}{2 s-1}}\right)<1-\frac{\sqrt{2}}{2} .
\end{aligned}
$$

Hence, from Proposition 6.2

$$
\begin{aligned}
\mu_{\text {norm }}^{m}\left(h, \zeta^{\prime}\right) & \leq \frac{(1-u)^{2}}{2 u^{2}-4 u+1} \mu_{\text {norm }}^{m}(h, \zeta) \\
& \leq \frac{\left(1-\left(1-\sqrt{\frac{s}{2 s-1}}\right)\right)^{2}}{2\left(1-\sqrt{\frac{s}{2 s-1}}\right)^{2}-4\left(1-\sqrt{\frac{s}{2 s-1}}\right)+1} \mu_{\text {norm }}^{m}(h, \zeta) \\
& \leq \frac{\frac{s}{2 s-1}}{\frac{1}{2 s-1}} \frac{1}{s \varepsilon}=\frac{1}{\varepsilon},
\end{aligned}
$$

which is false by hypothesis.

The following result is an upper bound for the probability distribution of the condition number $\mu_{\text {worst }}^{m}$ in $\mathbb{P}\left(\mathcal{H}_{(d)}^{m}\right)$.

Theorem 6.4. Let $0<\varepsilon<d^{3 / 2}$ be any positive number, and assume that $m<n$. Then, for a randomly chosen system $h \in \mathbb{P}\left(\mathcal{H}_{(d)}^{m}\right)$, the probability that $\mu_{\mathrm{worst}}^{m}(h)>$ $1 / \varepsilon$ is at most

$$
2 \mathcal{D}\left[10 m \sqrt{n N} d^{3 / 2}\right]^{2(n-m)}[6 m \sqrt{n N} \varepsilon]^{4} .
$$

Proof. Let $T_{\varepsilon} \subseteq \mathbb{P}\left(\mathcal{H}_{(d)}^{m}\right)$ be the set defined a follows:

$$
T_{\varepsilon}:=\left\{h \in \mathbb{P}\left(\mathcal{H}_{(d)}^{m}\right): \exists \zeta \in V_{\mathbb{P}}(h), \mu_{\text {norm }}^{m}(h, \zeta)>1 / \varepsilon\right\} .
$$

The probability of the theorem equals

$$
\frac{\nu\left[T_{\varepsilon}\right]}{\nu\left[\mathbb{P}\left(\mathcal{H}_{(d)}^{m}\right)\right]}=\frac{1}{\nu\left[\mathbb{P}\left(\mathcal{H}_{(d)}^{m}\right)\right]} \int_{h \in T_{\varepsilon}} 1 d \mathbb{P}\left(\mathcal{H}_{(d)}^{m}\right) .
$$

For every positive real number $s>1$, we define the following quantity:

$$
M I N_{\varepsilon, s}:=\min _{h \in T_{\varepsilon}} \nu\left[\zeta \in V_{\mathbb{P}}(h): \mu_{\text {norm }}^{m}(h, \zeta)>1 /(s \varepsilon)\right] .
$$

We will prove that $M I N_{\varepsilon, s}$ is a positive number for $s>1$. Hence, we have that

$$
\begin{gathered}
\frac{\nu\left[T_{\varepsilon}\right]}{\nu\left[\mathbb{P}\left(\mathcal{H}_{(d)}^{m}\right)\right]} \leq \frac{1}{\nu\left[\mathbb{P}\left(\mathcal{H}_{(d)}^{m}\right)\right] M I N_{\varepsilon, s}} \\
\times \int_{h \in T_{\varepsilon}} \nu\left[\zeta \in V_{\mathbb{P}}(h): \mu_{\text {norm }}^{m}(h, \zeta)>1 /(s \varepsilon)\right] d \mathbb{P}\left(\mathcal{H}_{(d)}^{m}\right) \\
\leq \frac{1}{\nu\left[\mathbb{P}\left(\mathcal{H}_{(d)}^{m}\right)\right] M I N_{\varepsilon, s}} \int_{h \in \mathbb{P}\left(\mathcal{H}_{(d)}^{m}\right)} \nu\left[\zeta \in V_{\mathbb{P}}(h): \mu_{\text {norm }}^{m}(h, \zeta)>1 /(s \varepsilon)\right] d \mathbb{P}\left(\mathcal{H}_{(d)}^{m}\right) .
\end{gathered}
$$


From Corollary 6.1 we have that

$$
\begin{aligned}
& \frac{1}{\nu\left[\mathbb{P}\left(\mathcal{H}_{(d)}^{m}\right)\right]} \int_{h \in \mathbb{P}\left(\mathcal{H}_{(d)}^{m}\right)} \nu\left[\zeta \in V_{\mathbb{P}}(h): \mu_{\text {norm }}^{m}(h, \zeta)>1 /(s \varepsilon)\right] d \mathbb{P}\left(\mathcal{H}_{(d)}^{m}\right) \\
& \leq \nu\left[\mathbb{P}_{n-m}(\mathbb{C})\right] \mathcal{D}[\operatorname{sem} \sqrt{n N} \varepsilon]^{2(n-m+2)}
\end{aligned}
$$

We conclude the following inequality:

$$
\frac{\nu\left[T_{\varepsilon}\right]}{\nu\left[\mathbb{P}\left(\mathcal{H}_{(d)}^{m}\right)\right]} \leq \frac{\nu\left[\mathbb{P}_{n-m}(\mathbb{C})\right] \mathcal{D}[\operatorname{sem} \sqrt{n N} \varepsilon]^{2(n-m+2)}}{M I N_{\varepsilon, s}},
$$

for every positive real number $s>1$. Now, we can give a lower bound for $M I N_{\varepsilon, s}$. In fact, let $h \in T_{\varepsilon}$ be a system, and let $\zeta^{\prime} \in V_{\mathbb{P}}(h)$ be such that $\mu_{\text {norm }}^{m}\left(h, \zeta^{\prime}\right)>1 / \varepsilon$. We may assume that every point of $V_{\mathbb{P}}(h)$ is a regular solution of $h$, as the set of systems not satisfying this hypothesis has measure zero in $\mathbb{P}\left(\mathcal{H}_{(d)}^{m}\right)$ and has no effect for integration purposes. Then, from Corollary 6.3, we have

$$
\nu\left[\zeta \in V_{\mathbb{P}}(h): \mu_{\mathrm{norm}}^{m}(h, \zeta)>\frac{1}{s \varepsilon}\right] \geq \nu\left[V_{\mathbb{P}}(h) \cap B_{\mathbb{P}}\left(\zeta^{\prime}, \frac{\sqrt{2} \varepsilon}{d^{3 / 2}} s\left(1-\sqrt{\frac{s}{2 s-1}}\right)\right)\right],
$$

where $B_{\mathbb{P}}(x, \lambda)$ is the ball in $\mathbb{P}_{n}(\mathbb{C})$ centered at $x$ of radius $\lambda$, for the projective distance $d_{\mathbb{P}}$. Moreover, $V_{\mathbb{P}}(h)$ is a smooth algebraic variety of complex dimension $n-m$. From [BP06a, Th. 24] we can give a lower bound estimation for this last quantity:

$$
\begin{gathered}
\nu\left[V_{\mathbb{P}}(h) \cap B_{\mathbb{P}}\left(\zeta^{\prime}, \frac{\sqrt{2} \varepsilon}{d^{3 / 2}} s\left(1-\sqrt{\frac{s}{2 s-1}}\right)\right)\right] \\
\geq \frac{1}{2} \nu\left[\mathbb{P}_{n-m}(\mathbb{C})\right]\left(\frac{\sqrt{2} \varepsilon}{d^{3 / 2}} s\left(1-\sqrt{\frac{s}{2 s-1}}\right)\right)^{2(n-m)},
\end{gathered}
$$

whenever the following inequality holds:

$$
\frac{\sqrt{2} \varepsilon}{d^{3 / 2}} s\left(1-\sqrt{\frac{s}{2 s-1}}\right) \leq \frac{\sqrt{2}}{2} .
$$

We conclude that, in this case,

$$
\operatorname{MIN}_{\varepsilon, s} \geq \frac{1}{2} \nu\left[\mathbb{P}_{n-m}(\mathbb{C})\right]\left(\frac{\sqrt{2} \varepsilon}{d^{3 / 2}} s\left(1-\sqrt{\frac{s}{2 s-1}}\right)\right)^{2(n-m)} .
$$

Finally, this implies the following inequality:

$$
\frac{\nu\left[T_{\varepsilon}\right]}{\nu\left[\mathbb{P}\left(\mathcal{H}_{(d)}^{m}\right)\right]} \leq 2 \mathcal{D}\left[\frac{e}{\sqrt{2}} m \sqrt{n N} d^{3 / 2}\right]^{2(n-m)}[e m \sqrt{n N} \varepsilon]^{4} \frac{s^{4}}{\left(1-\sqrt{\frac{s}{2 s-1}}\right)^{2(n-m)}},
$$

which holds for every positive number $s>1$. Let $s:=\frac{6}{e}>1$ be this positive number. Then, we have that

$$
\frac{\nu\left[T_{\varepsilon}\right]}{\nu\left[\mathbb{P}\left(\mathcal{H}_{(d)}^{m}\right)\right]} \leq 2 \mathcal{D}\left[\frac{e}{\sqrt{2}} m \sqrt{n N} d^{3 / 2}\right]^{2(n-m)}[6 m \sqrt{n N} \varepsilon]^{4} \frac{1}{\left(1-\sqrt{\frac{6 / e}{12 / e-1}}\right)^{2(n-m)}},
$$


and the theorem follows from the fact that

$$
\frac{e}{\sqrt{2}\left(1-\sqrt{\frac{6 / e}{12 / e-1}}\right)} \leq 10 .
$$

We have imposed the condition (6.1). Some elementary calculations show that it suffices that

$$
\varepsilon \leq d^{3 / 2} .
$$

\subsection{Proof of Theorem 1.3 .}

Proof. From inequality (2.3), the following chain of inequalities holds:

$$
\mathrm{E}_{\mathcal{P}_{(d)}^{m}}\left[\mu_{\text {worst }}^{m}\right]=\mathrm{E}_{\mathbb{P}\left(\mathcal{P}_{(d)}^{m}\right)}\left[\mu_{\text {worst }}^{m}\right] \leq \mathrm{E}_{\mathbb{P}\left(\mathcal{H}_{(d)}^{m}\right)}\left[\mu_{\text {worst }}^{m}\right] .
$$

Hence, we concentrate our efforts on the estimation of this last quantity.

First, assume that $n>m$. Let $t>1 / d^{3 / 2}$ be any positive real number. Then, from Theorem 6.4 we have that

$$
\begin{gathered}
\operatorname{Prob}\left[h \in \mathbb{P}\left(\mathcal{H}_{(d)}^{m}\right): \mu_{\text {worst }}^{m}(h)>t\right]=\operatorname{Prob}\left[h \in \mathbb{P}\left(\mathcal{H}_{(d)}^{m}\right): \mu_{\text {worst }}^{m}(h)>\frac{1}{1 / t}\right] \\
\leq 2 \mathcal{D}\left[10 m \sqrt{n N} d^{3 / 2}\right]^{2(n-m)}[6 m \sqrt{n N}]^{4} \frac{1}{t^{4}} .
\end{gathered}
$$

From Lemma 2.4 we obtain

$$
\mathrm{E}_{\mathbb{P}\left(\mathcal{H}_{(d)}^{m}\right)}\left[\mu_{\text {worst }}^{m}\right] \leq \frac{4}{3}(2 \mathcal{D})^{1 / 4}\left[10 m \sqrt{n N} d^{3 / 2}\right]^{\frac{n-m}{2}} 6 m \sqrt{n N} .
$$

Now, observe that

$$
\frac{4}{3} 2^{1 / 4} 6 \leq 10
$$

and the theorem follows in the case that $m<n$.

Finally, assume that $m=n$. This case has been studied by Shub and Smale in SS93b. However, we can follow our scheme of proof (which in the zero-dimensional case is essentially the same as theirs). Observe that in this case,

$$
\begin{gathered}
\operatorname{Prob}\left[h \in \mathbb{P}\left(\mathcal{H}_{(d)}^{m}\right): \mu_{\text {worst }}^{m}(h)>t\right]=\operatorname{Prob}\left[h \in \mathbb{P}\left(\mathcal{H}_{(d)}^{m}\right): \mu_{\text {worst }}^{m}(h)>\frac{1}{1 / t}\right] \\
\leq \frac{1}{\nu\left[\mathbb{P}\left(\mathcal{H}_{(d)}^{m}\right)\right]} \int_{h \in \mathbb{P}\left(\mathcal{H}_{(d)}^{m}\right)} \sharp\left[\zeta \in V_{\mathbb{P}}(h): \mu_{\text {norm }}^{m}(h, \zeta)>\frac{1}{1 / t}\right] d \mathbb{P}\left(\mathcal{H}_{(d)}^{m}\right) .
\end{gathered}
$$

From Corollary 6.1 this last is at most

$$
\nu\left[\mathbb{P}_{0}(\mathbb{C})\right] \mathcal{D}\left(e n \sqrt{n N} \frac{1}{t}\right)^{4}=\mathcal{D}\left(e n \sqrt{n N} \frac{1}{t}\right)^{4} .
$$

From Lemma 2.4 this implies

$$
\mathrm{E}_{\mathbb{P}\left(\mathcal{H}_{(d)}^{m}\right)}\left[\mu_{\text {worst }}^{m}\right] \leq \frac{4}{3} \mathcal{D}^{1 / 4} e n \sqrt{n N} .
$$

In particular, the theorem holds, as $\frac{4}{3} e \leq 10$. 
6.2. Proof of Theorem 1.2. Observe that claim (1) in this result is a direct consequence of claim (2). Moreover, claim (3) is also a consequence of claim (2). In fact, the Jensen inequality yields

$$
\mathrm{E}\left[\frac{1}{X}\right] \geq \frac{1}{\mathrm{E}[X]}
$$

for any random variable $X$. Now, from Corollary 1.1 the convergence radius for $f \in \mathcal{P}_{(d)}$ is at least $u_{0} \gamma_{\text {worst }}(f)^{-1}$. Hence, it suffices to prove claim (2). But claim (2) is immediate from Proposition 3.4 and Theorem 1.3.

\section{REFERENCES}

[BCSS98] L. Blum, F. Cucker, M. Shub, and S. Smale, Complexity and real computation, Springer-Verlag, New York, 1998. MR.1479636 (99a:68070)

[Bel06] C. Beltrán, Sobre el Problema 17 de Smale: Teoría de la Intersección y Geometría Integral, Ph.D. Thesis., Universidad de Cantabria, 2006.

[BP05] C. Beltrán and L.M. Pardo, Upper bounds on the distribution of the condition number of singular matrices, C. R. Math. Acad. Sci. Paris 340 (2005), no. 12, 915-919. MR 2152279

[BP06a] C. Beltrán and L.M. Pardo, Estimates on the distribution of the condition number of singular matrices., Found. Comput. Math. To appear (2006).

[BP06b] On Smale's 17th problem: A probabilistic positive answer, Found. Comput. Math. To appear (2006).

[Ded97] J.P. Dedieu, Estimations for the separation number of a polynomial system, J. Symbolic Comput. 24 (1997), no. 6, 683-693. MR.1487794(99b:65065)

[Ded01] - Newton's method and some complexity aspects of the zero-finding problem, Foundations of Computational Mathematics (Oxford, 1999), London Math. Soc. Lecture Note Ser., vol. 284, Cambridge Univ. Press, Cambridge, 2001, pp. 45-67. MR 1836614 (2002d:65050)

[Ded06] Points fixes, zéros et la méthode de Newton, Collection Mathématiques et Applications, Springer, to appear 2006.

[Dég01] J. Dégot, A condition number theorem for underdetermined polynomial systems, Math. Comp. 70 (2001), no. 233, 329-335. MR.1458220 (2001f:65060)

[Dem88] J. W. Demmel, The probability that a numerical analysis problem is difficult, Math. Comp. 50 (1988), no. 182, 449-480. MR.929546 (89g:65062)

[DS01] J.P. Dedieu and M. Shub, On simple double zeros and badly conditioned zeros of analytic functions of $n$ variables, Math. Comp. 70 (2001), no. 233, 319-327. MR1680867 (2001f:65033)

[EGP00] N. Elezović, C. Giordano, and J. Pečarić, The best bounds in Gautschi's inequality, Math. Inequal. Appl. 3 (2000), no. 2, 239-252. MR.1749300 (2001g:33001)

[Fed69] H. Federer, Geometric measure theory, Die Grundlehren der mathematischen Wissenschaften, Band 153, Springer-Verlag New York Inc., New York, 1969. MR0257325 (41:1976)

[GLSY05] M. Giusti, G. Lecerf, B. Salvy, and J.P. Yakoubsohn, On location and approximation of clusters of zeros: case of embedding dimension one, Found. Comp. Mathematics, to appear (2005).

[GVL96] Gene H. Golub and Charles F. Van Loan, Matrix computations, third ed., Johns Hopkins Studies in the Mathematical Sciences, Johns Hopkins University Press, Baltimore, MD, 1996. MR 1417720 (97g:65006)

[Hei83] J. Heintz, Definability and fast quantifier elimination in algebraically closed fields, Theoret. Comput. Sci. 24 (1983), no. 3, 239-277. MR716823 (85a:68062)

[Hig02] N.J. Higham, Accuracy and stability of numerical algorithms, second ed., Society for Industrial and Applied Mathematics (SIAM), Philadelphia, PA, 2002. MR1927606 (2003g:65064)

[Kah00] W. Kahan, Huge generalized inverses of rank-deficient matrices., Unpublished Manuscript, 2000. 
[Kim89] M.H. Kim, Topological complexity of a root finding algorithm, J. Complexity 5 (1989), no. 3, 331-344. MR.1018023 (90m:65058)

[Kun85] E. Kunz, Introduction to commutative algebra and algebraic geometry, Birkhäuser Boston Inc., Boston, MA, 1985. MR.789602 (86e:14001)

[Mal94] G. Malajovich, On generalized Newton algorithms: Quadratic convergence, pathfollowing and error analysis, Theoret. Comput. Sci. 133 (1994), no. 1, 65-84, Selected papers of the Workshop on Continuous Algorithms and Complexity (Barcelona, 1993). MR:1294426 (95g:65073)

[Mum76] D. Mumford, Algebraic geometry. I, Springer-Verlag, Berlin, 1976, Complex projective varieties, Grundlehren der Mathematischen Wissenschaften, No. 221. MR0453732 (56:11992)

[NG47] J. von Neumann and H. H. Goldstine, Numerical inverting of matrices of high order, Bull. Amer. Math. Soc. 53 (1947), 1021-1099. MR0024235 (9,471b)

[SS90] G. W. Stewart and J. G. Sun, Matrix perturbation theory, Computer Science and Scientific Computing, Academic Press Inc., Boston, MA, 1990. MR.1061154(92a:65017)

[SS93a] M. Shub and S. Smale, Complexity of Bézout's theorem. I. Geometric aspects, J. Amer. Math. Soc. 6 (1993), no. 2, 459-501. MR.1175980 (93k:65045)

[SS93b] Complexity of Bezout's theorem. II. Volumes and probabilities, Computational Algebraic Geometry (Nice, 1992), Progr. Math., vol. 109, Birkhäuser Boston, Boston, MA, 1993, pp. 267-285. MR.1230872 (94m:68086)

[SS93c] Complexity of Bezout's theorem. III. Condition number and packing, J. Complexity 9 (1993), no. 1, 4-14, Festschrift for Joseph F. Traub, Part I. MR 1213484 (94g:65152)

[SS94] Complexity of Bezout's theorem. V. Polynomial time, Theoret. Comput. Sci. 133 (1994), no. 1, 141-164, Selected papers of the Workshop on Continuous Algorithms and Complexity (Barcelona, 1993). MR1294430 (96d:65091)

[SS96] _ Complexity of Bezout's theorem. IV. Probability of success; extensions, SIAM J. Numer. Anal. 33 (1996), no. 1, 128-148. MR1377247 (97k:65310)

[TB97] L.N. Trefethen and D. Bau, III, Numerical linear algebra, Society for Industrial and Applied Mathematics (SIAM), Philadelphia, PA, 1997. MR.1444820 (98k:65002)

[Tur48] A. M. Turing, Rounding-off errors in matrix processes, Quart. J. Mech. Appl. Math. 1 (1948), 287-308. MR0028100 (10:405c)

[Wil65] J. H. Wilkinson, The algebraic eigenvalue problem, Clarendon Press, Oxford, 1965. MR.0184422(32:1894)

Departamento de Matemáticas, Estadística y Computación, Facultad de Ciencias, Universidad de Cantabria, E-39071 Santander, Spain

E-mail address: beltranc@unican.es

Departamento de Matemáticas, Estadística y Computación, Facultad de Ciencias, Universidad de Cantabria, E-39071 Santander, Spain

E-mail address: luis.pardo@unican.es 